\title{
Minimal Degree Coprime Factorization of Rational Matrices
}

\author{
Cristian OARĂ ${ }^{1}$ and Andras VARGA \\ German Aerospace Center \\ DLR - Oberpfaffenhofen \\ Institute of Robotics and System Dynamics \\ D-82234 Wessling, Germany \\ Emails: oara@df .op.dlr.de, Andras.Varga@dlr.de
}

\begin{abstract}
For a given rational matrix $G$ with complex coefficients and a given domain $\Gamma$ in the closed complex plane, both arbitrary, we develop a complete theory of coprime factorizations of $G$ over $\Gamma$, with denominators of McMillan degree as small as possible. The main tool is a general pole displacement theorem which gives conditions for an invertible rational matrix to dislocate by multiplication a part of the poles of $G$. We apply this result to obtain the parametrized class of all coprime factorizations over $\Gamma$ with denominators of minimal McMillan degree $n_{b}$ - the number of poles of $G$ outside $\Gamma$. Specific choices of the parameters and of $\Gamma$ allow to determine coprime factorizations, as for instance, with polynomial, proper or stable factors. Further, we consider the case in which the denominator has a certain symmetry, namely it is $J$ all-pass, either with respect to the imaginary axis or to the unit circle. We give necessary and sufficient solvability conditions for the problem of coprime factorization with $J$ all-pass denominator of McMillan degree $n_{b}$ and, when a solution exists, we give a construction of the class of coprime factors. When no such solution exists, we discuss the existence of and give solutions to coprime factorizations with $J$ all-pass denominators of minimal McMillan degree $\left(>n_{b}\right)$. All the developments are carried out in terms of descriptor realizations associated with rational matrices, leading to explicit and computationally efficient formulas.

Keywords: Rational Matrices; Coprime factorizations; $J$ all-pass; Descriptor realizations; Pole assignment; Numerical algorithms.
\end{abstract}

\section{Introduction}

Let $G$ be an arbitrary rational matrix (possible improper) and let $\Gamma$ be a given domain of the closed complex plane. A left coprime factorization (LCF) over $\Gamma$ of $G$ is a fractional representation of the form $G=M^{-1} N$, with $N$ and $M$ rational matrices having poles only in $\Gamma$ and satisfying $M U+N V=I$ for certain rational matrices $U$ and $V$ with all poles in $\Gamma$. Analogously, a factorization in the form $G=N M^{-1}$, with $N$ and $M$ having poles only in $\Gamma$, and satisfying $U M+V M=I$ for certain rational matrices $U$ and $V$ with poles in $\Gamma$ is called a right coprime factorization over $\Gamma$.

In this paper we develop a complete theory of LCFs over $\Gamma$ with denominators $M$ of smallest possible McMillan degree (see the precise definition below). For brevity, we call such factorizations of minimal degree, and define the degree of the factorization to be the McMillan degree of the denominator $M$. We address only LCFs since all results for right coprime factorizations follow by duality.

Apparently, the theory of minimal degree coprime factorizations has not been previously considered as such in the literature, although it is crux to solving various problems encountered in the

\footnotetext{
${ }^{1}$ The work of Cristian Oară was completed with the support of

Alexander von Humboldt Foundation, Bonn, Germany
} 
theory of linear systems and networks [24, 27], to canonical and noncanonical spectral factorizations of unstable rational matrices [3], to several conjugation based approaches to nonstandard $H_{\infty}$ control problems $[25,26]$, and brings important numerical advantages as for instance in computing coprime factorizations with denominators which are either polynomial, proper, stable, all-pass, or $J$ lossless $[19,20]$. The only noticeable exception is [2], where coprime factorizations are addressed with another requirement of minimality, namely the sum of the McMillan degrees of the denominator and nominator to be as small as possible. However, even in the quite particular case treated in [2] where the matrix to be factored is square and of full rank over rationals this sum-minimality leads to combinatorial problems over an infinite set which have little interest from an algorithmic viewpoint as well as for the type of applications that we have mentioned above.

Our main tool in the derivations - which has also an independent interest - is a general pole displacement theorem which gives a characterization in terms of realizations for an invertible rational matrix $M$ to cancel in the product $M G$ all poles of $G$ in $\Gamma$. An important feature of our result is the possibility to cope with domains containing infinity, without using conformal mapping techniques. In particular, we show that the minimal degree of a LCF over $\Gamma$ is $n_{b}$, where $n_{b}$ is the number of poles of $G$ outside $\Gamma$, and give a description in terms of realizations of all factors solving the minimal degree LCF over $\Gamma$. It turns out that the basic ingredient in computing LCFs of minimal degree lies in the solution of a generalized eigenvalue assignment problem of order $n_{b}$.

We develop also a theory of minimal degree LCFs with the additional requirement that the denominator has a certain symmetry. We consider here two cases, in which the denominator is $J$ all-pass, either with respect to the imaginary axis or to the unit circle, and the domain $\Gamma$ is adequately chosen to reflect the respective symmetry. We study first LCFs with $J$ all-pass denominator of degree $n_{b}$ which we call the canonical case, and give necessary and sufficient solvability conditions. When solutions exist we give a parametrized description of the factors in terms of associated realizations. When such canonical solutions do not exist, we discuss the solution of noncanonical LCFs with $J$ all-pass denominators of McMillan degree as small as possible $\left(>n_{b}\right)$. The computation of factors in the canonical case relies on solving a generalized Lyapunov equation of order $n_{b}$. In the noncanonical case, the rank $r$ of the solution of the same Lyapunov equation allows to determine the minimal degree of the factorization as $2 n_{b}-r$. The noncanonical factors can then be constructed by embedding the solution of this Lyapunov equation of order $n_{b}$ into a $2 n_{b}-r$ order Lyapunov equation with nonsingular solution. The noncanonical case treated here has important connections with the noncanonical Wiener-Hopf and $J$ spectral factorizations as discussed in [3] for proper and invertible rational matrices. Another connected work is [26] which in contrast to our results is restricted to the canonical case with $J$ lossless denominator with respect to the imaginary axis, and involves the solution of a Riccati equation of order equal to the McMillan degree of $G$.

The paper is organized as follows. The main definitions and notation used throughout the paper are presented in a preliminary section together with a brief discussion on various descriptor realizations of rational matrices. In Section 3 we obtain formnlas for an invertible factor that dislocates by left multiplication certain prescribed poles of a given rational matrix. In Section 4 we give a parametrization of the class of factors that solve the minimal degree LCF over $\Gamma$. Section 5 is devoted to the theory of minimal degree LCFs with $J$ all-pass denominators with respect to the imaginary axis. In Section 6 we develop the analogous results in the case in which the denominator is $J$ all-pass with respect to the unit circle. Several numerical examples are presented in Section 7 together with a discussion of appropriate computational methods. Some conclusions summarize the main aspects of the proposed theory and provide hints to possible applications and extensions. We defer to an Appendix the characterization in terms of realizations of an arbitrary $J$ all-pass rational matrix.

\section{Preliminaries}

\subsection{Basic notation}

We start with some notation and definitions. For a matrix $A$ we denote by $A^{T}$ and $A^{*}$, its transpose and its conjugate transpose, respectively, and if $A$ is invertible by $A^{-T}$ and $A^{-*}$ its transposed 
inverse and its conjugated transposed inverse, respectively.

By $\mathbb{C}, \mathbb{C}^{-}, \mathbb{C}^{+}, \mathbb{C}^{0}$, and $\mathbb{R}$ we denote the complex plane, the open left half plane, the open right half plane, the imaginary axis, and the real axis, respectively, and let $\overline{\mathbb{C}}:=\mathbb{C} \cup\{\infty\}$ be the closed complex plane, and $\overline{\mathbb{C}}^{-}:=\mathbb{C}^{-} \cup \mathbb{C}^{0} \cup\{\infty\}, \overline{\mathbb{C}}^{+}:=\mathbb{C}^{+} \cup \mathbb{C}^{0} \cup\{\infty\}, \overline{\mathbb{C}}^{0}:=\mathbb{C}^{0} \cup\{\infty\}$. Here "overbar" denotes closure. By $\mathbb{D}$ we denote the open unit disk and $\mathbb{D}^{c}=\overline{\mathbb{C}} \backslash \overline{\mathbb{D}}$ stands for the exterior of the closed unit disk, containing the infinity.

Consider the disjoint partition of the closed complex plane into a "good" region $\Gamma_{g}$ and a "bad" region $\Gamma_{b}$ as

$$
\overline{\mathbf{C}}=\Gamma_{g} \cup \Gamma_{b} .
$$

A frequent interpretation of $\Gamma_{g}$ in system theory is related to the standard stability concept, that is, for linear continuous-time systems $\Gamma_{g}=\mathbb{C}^{-}$, while for linear discrete-time systems $\Gamma_{g}=\mathbb{D}$ (or, sometimes, their closures). However, we use also other interpretations, as for instance $\Gamma_{g}=\{\infty\}$ to obtain coprime factorizations with polynomial factors. When dealing with LCFs of rational matrices with real coefficients, we assume that $\Gamma_{g}$ is symmetric with respect to the imaginary axis, that is, if $\lambda \in \Gamma_{g}$ then $\bar{\lambda} \in \Gamma_{g}$. This is in particular true for all the aforementioned examples of $\Gamma_{g}$ and guarantees that the resulting factors of the LCF have real coefficients as well.

\subsection{Rational matrices}

Throughout the paper we consider matrices with coefficients in the field $\mathbb{F}$, where $\mathbb{F}$ denotes either $\mathbb{R}$ or $\mathbb{C}$. We view a $p \times m$ rational matrix $G(\lambda)$ as a matrix whose entries are rational functions with coefficients in $\mathbb{E}$ and of the complex variable $\lambda$. Alternatively, we shall assimilate the rational matrix $G(\lambda)$ with the transfer-function matrix (TFM) of a linear time-invariant continuous-time or discrete-time descriptor system, and $\lambda$ is either $s$ or $z$, the complex variables appearing in the Laplace- or Z-transform, respectively, according to the type of the system. We denote with $G^{\sim}$ the adjoint of $G$, where $G^{\sim}(s)=G^{*}(-\bar{s})$ in continuous-time and $G^{\sim}(z)=G^{*}(1 / \bar{z})$ in discrete-time. In particular, if $G$ has real coefficients we have $G^{\sim}(s)=G^{T}(-s)$ and $G^{\sim}(z)=G^{T}(1 / z)$, respectively. Let $J$ be a signature matrix, i.e., a matrix satisfying $J=J^{*}=J^{-1}$. We say that the rational matrix $G$ is $J$ all-pass with respect to the imaginary axis if $G^{\sim} J G=J$, where $G^{\sim}$ denotes the adjoint in continuous-time. Accordingly, we say that $G$ is $J$ all-pass with respect to the unit circle if $G^{\sim} J G=J$, where $G^{\sim}$ denotes now the adjoint in discrete-time.

\subsection{Structural elements of rational matrices}

For an arbitrary rational matrix we recall now several structural elements that play an important role in the sequel: normal rank, finite and infinite poles and zeros, and their orders. It is easy to realize that $G(\lambda)$ has constant rank for all but a finite number of points $\lambda \in \mathbb{C}$ for which it has smaller rank. The normal rank of $G$ is by definition the rank of $G(\lambda)$ for almost all $\lambda \in \mathbb{C}$. A rational matrix which is square (say $p \times p$ ) and has full normal rank $p$ is called nonsingular, otherwise it is called singular. Clearly, a nonsingular rational matrix has an inverse which is a rational matrix as well.

In this paper, a particular focus is on the poles and zeros of a rational matrix, defined now. For an arbitrary $p \times m$ rational matrix $G$ of normal rank $r$ there always exist a $p \times p$ polynomial matrix $U(\lambda)$ with $\operatorname{det} U(\lambda)=1$, and an $m \times m$ polynomial matrix $V(\lambda)$ with $\operatorname{det} V(\lambda)=1$ that bring $G$ to the so-called Smith-McMillan form

$$
D(\lambda)=U(\lambda) G(\lambda) V(\lambda)
$$

where

$$
D(\lambda)=\operatorname{diag}\left\{\frac{\epsilon_{1}(\lambda)}{\eta_{1}(\lambda)}, \frac{\epsilon_{2}(\lambda)}{\eta_{2}(\lambda)}, \ldots, \frac{\epsilon_{r}(\lambda)}{\eta_{r}(\lambda)}, 0, \ldots, 0\right\},
$$

the polynomials $\epsilon_{i}(\lambda), \eta_{i}(\lambda)$ are monic (have the highest degree coefficient equal to 1 ), are pairwise coprime for $i=1, \ldots, r$, and satisfy the divisibility properties

$$
\begin{array}{r|l}
\epsilon_{i}(\lambda) & \epsilon_{i+1}(\lambda), \quad i=1, \ldots, r-1 \\
\eta_{i+1}(\lambda) & \eta_{i}(\lambda)
\end{array}
$$


The polynomials $\epsilon_{i}(\lambda)$ and $\eta_{i}(\lambda)$ are uniquely defined by $G(\lambda)$, and are called elementary divisors of $G$. Define

$$
\begin{aligned}
& z(\lambda)=\epsilon_{1}(\lambda) \cdots \epsilon_{r}(\lambda), \\
& p(\lambda)=\eta_{1}(\lambda) \cdots \eta_{r}(\lambda) .
\end{aligned}
$$

Then the $n_{z}\left(n_{p}\right)$ roots of $z(\lambda)(p(\lambda))$ are by definition the finite zeros (poles) of $G$ and their multiplicities are by definition the multiplicities of the roots of $z(\lambda)(p(\lambda))$. The orders of the zero (pole) $\lambda_{0}$ of $G$ are by definition the nonzero multiplicities of $\lambda_{0}$ as a root of $\epsilon_{i}(\lambda)\left(\eta_{i}(\lambda)\right)$, for $i=1, \ldots, r$. We say that $\infty$ is a zero (pole) of $G$ if 0 is a zero (pole) of the rational matrix $\widehat{G}(\lambda)=G\left(\frac{1}{\lambda}\right)$, and we define the orders of the (zero) pole $\lambda=\infty$ of $G(\lambda)$ as the orders of the pole $\lambda=0$ of $\widehat{G}(\lambda)$.

By definition, the McMillan degree of $G$ - denoted $\delta(G)$ - is the sum of the orders of all its poles (finite and infinite). Once a partition (1) is fixed, we have $\delta(G)=n_{b}+n_{g}$, where $n_{g}$ denotes the number of "good" poles in $\Gamma_{g}$ and $n_{b}$ denotes the number of "bad" poles in $\Gamma_{\bar{b}}$ (counting multiplicities).

A rational matrix $G$ which is $J$ all-pass with respect to the imaginary axis features a pole-zero symmetry with respect to the imaginary axis. Precisely, if $\left\{\lambda_{1}, \lambda_{2}, \ldots, \lambda_{n}\right\}$ is the union of poles of $G$, then $\left\{-\bar{\lambda}_{1},-\bar{\lambda}_{2}, \ldots,-\bar{\lambda}_{n}\right\}$ is the union of zeros of $G$. A similar property holds for a rational matrix $G$ which is $J$ all-pass with respect to the unit circle where now the pole-zero symmetry is of course with respect to the unit circle.

\subsection{Descriptor realizations of rational matrices}

\section{Standard descriptor realizations of rational matrices}

It is well known (see for example $[23,22]$ ) that any $p \times m$ rational matrix $G(\lambda)$ with coefficients in $\mathbb{F}$ (even improper or polynomial) has a descriptor realization of the form

$$
G(\lambda)=D+C(\lambda E-A)^{-1} B=:\left[\begin{array}{c|c}
A-\lambda E & B \\
\hline C & D
\end{array}\right],
$$

where $A, E \in \mathbb{F}^{n \times n}, B \in \mathbb{F}^{n \times m}, C \in \mathbb{F}^{p \times n}, D \in \mathbb{F}^{p \times m}$, and the so called pole pencil $A-\lambda E$ is regular, i.e., it is square and $\operatorname{det}(A-\lambda E) \not \equiv 0$. The dimension $n$ of the square matrices $A$ and $E$ is called the order of the realization $(6)$. We use $\Lambda(A-\lambda E)$ to denote the union of generalized eigenvalues of the regular pencil $A-\lambda E$ (finite and infinite, multiplicities counting). Occasionally, we shall also use the more compact notation $G=(A-\lambda E, B, C, D)$ to denote (6). The descriptor realization (6) of $G$ is called minimal if its order is as small as possible among all realizations of this kind. Well known criteria for minimality of a descriptor realization (6) are (see for example [22]):

$$
\begin{aligned}
& \operatorname{rank}\left[\begin{array}{cc}
A-\lambda E & B
\end{array}\right]=n, \quad \forall \lambda \in \mathbb{C}, \\
& \operatorname{rank}\left[\begin{array}{cc}
E & B
\end{array}\right]=n, \\
& \operatorname{rank}\left[\begin{array}{c}
A-\lambda E \\
C
\end{array}\right]=n, \quad \forall \lambda \in \mathbb{C}, \\
& \operatorname{rank}\left[\begin{array}{c}
E \\
C
\end{array}\right]=n, \\
& A \operatorname{ker}(E) \subseteq \operatorname{Im}(E) .
\end{aligned}
$$

The conditions of minimality (i)-(v) are usually known as finite and infinite controllability, finite and infinite observability, and absence of nondynamic modes, respectively. For a minimal descriptor realization (6) of order $n$ we have $\delta(G)=\operatorname{rank} E \leq n$.

The principal inconvenience of realizations of the form (6) is that their minimal possible order is greater than the McMillan degree of $G$, unless $G$ is proper, and this brings important technical difficulties in factorization problems in which the McMillan degree plays a paramount role. A remedy to this is to use a generalization of $(6)$ in which either the " $B$ " or the " $C$ " matrix is replaced by a matrix pencil, as explained in the next section. 


\section{Alternative descriptor realizations}

Any rational $p \times m$ matrix $G$ has a realization

$$
G(\lambda)=D+C(\lambda E-A)^{-1}(B-\lambda F)=:\left[\begin{array}{c|c}
A-\lambda E & B-\lambda F \\
\hline C & D
\end{array}\right],
$$

and for any fixed $\alpha, \beta \in \mathbb{F}$, not both zero, there exists a realization

$$
G(\lambda)=D+C(\lambda E-A)^{-1} B(\alpha-\lambda \beta)=:\left[\begin{array}{c|c}
A-\lambda E & B(\alpha-\lambda \beta) \\
\hline C & D
\end{array},\right.
$$

where $A, E \in \mathbb{F}^{n \times n}, B, F \in \mathbb{F}^{n \times m}, C \in \mathbb{F}^{p \times n}, D \in \mathbb{F}^{p \times m}$, and the pole pencil $A-\lambda E$ is regular. A realization (9) will be called centered at $\frac{\alpha}{\beta}$ (if $\beta=0$ we interpret $\frac{\alpha}{\beta}$ as $\infty$ ). Occasionally, we shall use also the more compact notation $G=(A-\lambda E, B-\lambda F, C, D)$ and $G=(A-\lambda E, B(\alpha-\lambda \beta), C, D)$ to denote (8) and (9), respectively.

All realizations (6), (8) and (9) are formally contained in the following general reatization of a rational matrix due to Rosenbrock [14]

$$
G(\lambda)=W(\lambda)-V(\lambda) T^{-1}(\lambda) U(\lambda)=:\left[\begin{array}{c|c}
T(\lambda) & U(\lambda) \\
\hline V(\lambda) & W(\lambda)
\end{array}\right]
$$

where $T(\lambda), U(\lambda), V(\lambda)$, and $W(\lambda)$ are matrix polynomials, with $T(\lambda)$ square and invertible. It is easy to see that

$$
\left[\begin{array}{cc}
\widetilde{T}(\lambda) & \widetilde{U}(\lambda) \\
\widetilde{V}(\lambda) & \widetilde{W}(\lambda)
\end{array}\right]=\left[\begin{array}{ll}
Q & O \\
X & I
\end{array}\right]\left[\begin{array}{cc}
T(\lambda) & U(\lambda) \\
V(\lambda) & W(\lambda)
\end{array}\right]\left[\begin{array}{cc}
Z & Y \\
O & I
\end{array}\right]
$$

where $Q$ and $Z$ are constant invertible matrices, and $X$ and $Y$ are arbitrary constant matrices, defines an equivalent realization in the form

$$
G(\lambda)=\left[\begin{array}{c|c}
\widetilde{T}(\lambda) & \widetilde{U}(\lambda) \\
\hline \widetilde{V}(\lambda) & \widetilde{W}(\lambda)
\end{array}\right]
$$

(see also [14] for a more general case). This type of transformation performed on a realization is called equivalence, and if in addition $X=0$ and $Y=0$ it is called state-space equivalence.

We call realizations of the type (8) or (9) minimal if the dimension of the square matrices $A$ and $E$ (also called the order of the realization) is as small as possible among all realizations of the respective kind. It can be easily shown that any rational matrix $G(\lambda)$ has a minimal realization of type (8) of order equal to $\delta(G)$. For any fixed $\alpha$ and $\beta$, not both zero, and such that $\frac{\alpha}{\beta}$ is not a pole of $G$ there also exists a minimal realization of type (9) of order equal to $\delta(G)$. The condition imposed on $\frac{\alpha}{\beta}$ is needed only for writing down minimal realizations (9) which have order equal to $\delta(G)$. More precisely, even if $\frac{\alpha}{\beta}$ is a pole of $G$ we can still write a realization (9) but the minimal order will with necessity be greater than $\delta(G)$. This is exactly what is happening for realizations (6) which are obtained from (9) for $\alpha=1$ and $\beta=0$, and for which the minimal order is necessary greater than $\delta(G)$, provided $\frac{\alpha}{\beta}=\infty$ is a pole of $G$. Notice that for (9) we can always choose freely $\alpha$ and $\beta$ such as to ensure $\frac{\alpha}{\beta}$ is not a pole of $G$. For the rest of the paper, if not otherwise stated, we assume this choice implicitly. The nice feature of (8) and (9) that their minimal order equals the McMillan degree of $G$ recommends them for the kind of problems treated in this paper.

It is relatively straightforward to show that the criteria of minimality for a descriptor realization of type (8) are

$$
\begin{aligned}
& \operatorname{rank}\left[\begin{array}{cc}
A-\lambda E & B-\lambda F
\end{array}\right]=n, \quad \forall \lambda \in \mathbb{C}, \\
& \operatorname{rank}\left[\begin{array}{ll}
E & F
\end{array}\right]=n \text {, } \\
& \operatorname{rank}\left[\begin{array}{c}
A-\lambda E \\
C
\end{array}\right]=n, \quad \forall \lambda \in \mathbb{C}, \\
& \operatorname{rank}\left[\begin{array}{c}
E \\
C
\end{array}\right]=n
\end{aligned}
$$


while for realizations of type (9) similar conditions result by simply replacing (i) and (ii) in (12) with

$$
\begin{aligned}
& \operatorname{rank}\left[\begin{array}{ll}
A-\lambda E & B(\alpha-\lambda \beta)
\end{array}\right]=n, \quad \forall \lambda \in \mathbb{C}, \\
& \operatorname{rank}\left[\begin{array}{ll}
E & B
\end{array}\right]=n .
\end{aligned}
$$

Of particular importance in the case of (9) is that any two minimal realizations $G=(A-\lambda E, B(\alpha-$ $\lambda \beta), C, D)$ and $G=(\widetilde{A}-\lambda \widetilde{E}, \widetilde{B}(\alpha-\lambda \beta), \widetilde{C}, \widetilde{D})$ are always related by an equivalence transformation as

$$
\widetilde{E}=Q E Z, \quad \widetilde{A}=Q A Z, \quad \widetilde{B}=Q B, \quad \widetilde{C}=C Z, \quad \widetilde{D}=D,
$$

where $Q$ and $Z$ are unique invertible matrices. Moreover, for an invertible rational matrix $G(\lambda)$, it is possible to choose a realization (9) with $D$ invertible, provided $\alpha$ and $\beta$ are such that $\frac{\alpha}{\beta}$ is neither a pole nor a zero of $G(\lambda)$. In this case we can use explicit formulas for the inverse $G^{-1}$ in the form

$$
G^{-1}(\lambda)=\left[\begin{array}{c|c}
A-\lambda E-B D^{-1} C(\alpha-\lambda \beta) & B D^{-1}(\alpha-\lambda \beta) \\
\hline-D^{-1} C
\end{array}\right] .
$$

An additional nice feature of realizations (9) is revealed in Appendix where we show that an appropriate choice of $\alpha$ and $\beta$ leads to nice formulas in case of rational matrices featuring certain symmetries.

Conversions between the representations (6), (8) and (9) are straightforward. Here we only show how to obtain from a $n$-th order realization (6) a realization of the form (8) of order equal to rank $E$. We can always determine two invertible (even unitary) matrices $Q$ and $Z$ such that

$$
G(\lambda)=\left[\begin{array}{c|c}
Q(A-\lambda E) Z & Q B \\
\hline C Z & \bar{D}
\end{array}\right]=\left[\begin{array}{cc|c}
A_{d}-\lambda E_{d} & A_{d n}-\lambda E_{d n} & B_{d} \\
O & A_{n} & B_{n} \\
\hline C_{d} & C_{n} & \bar{D}
\end{array}\right],
$$

where the invertible matrix $A_{n} \in \mathbb{R}^{\kappa \times \kappa}$ contains the so-called infinite nondynamic modes, and $\operatorname{rank}\left[\begin{array}{ll}E_{d} & E_{d n}\end{array}\right]=\operatorname{rank} E$. By using a general transformation as in (11) we get an equivalent realization of the type (8) in the form

$$
G(\lambda)=\left[\begin{array}{c|c}
A_{d}-\lambda E_{d} & B_{d}-A_{d n} A_{n}^{-1} B_{n}+\lambda E_{d n} A_{n}^{-1} B_{n} \\
\hline C_{d} & D-C_{n} A_{n}^{-1} B_{n}
\end{array}\right]
$$

which has order equal to rank $E$. Provided (6) is minimal, the realization (16) is minimal as well.

All the above stated features of realizations (8) and (9) although not well-known, follow by standard arguments from the realization theory $[14,22]$, and therefore we do not give here detailed proofs.

\section{Structural elements in terms of realizations}

The following result adapted from [14] and [23] will be instrumental in the sequel.

Theorem 2.1 Let $G(\lambda)$ be an arbitrary rational matrix with a realization (8) (or (9)).

1. Poles. The poles of $G(\lambda)$ are among the generalized eigenvalues of the pole pencil $A-\lambda E$. If the realization (8) is minimal (or if $\frac{\alpha}{\mathcal{G}}$ is not a pole of $G(\lambda)$, and (9) is minimal) then the poles of $G(\lambda)$ are exactly the generalized eigenvalues of $A-\lambda E$, and the orders of the poles are pairwise equal to the multiplicities of the generalized eigenvalues of $A-\lambda E$.

2. Zeros. The zeros of $G(\lambda)$ are among the zeros of the system pencil associated with (8)

$$
\left[\begin{array}{cc}
A-\lambda E & B-\lambda F \\
C & D
\end{array}\right],
$$

(or of the system pencil associated with (9)

$$
\left[\begin{array}{cc}
A-\lambda E & B(\alpha-\lambda \beta) \\
C & D
\end{array}\right]
$$


For a careful discussion of the delicate notions of pole and zero at infinity of a rational matrix and connection with the realization theory we refer to [23].

\section{Separated realizations}

As a methodological question, throughout the paper we assume that the rational matrix $G$ to start with is given by a minimal descriptor realization (6) as this type of realization is most frequently used in the literature to represent arbitrary (possibly improper) rational matrices. The solutions $M$ and $N$ to the LCF $G=M^{-1} N$ will be given directly by minimal realizations of the type (8) or (9) of order equal to their respective McMillan degree. Furthermore, once a partition (1) is fixed, we assume that $G$ is given by a separated realization with respect to (1), namely

$$
G(\lambda)=\left[\begin{array}{cc|c}
A_{b}-\lambda E_{b} & A_{b g}-\lambda E_{b g} & B_{b} \\
O & A_{g}-\lambda E_{g} & B_{g} \\
\hline C_{b} & C_{g} & D
\end{array}\right]
$$

where the $n_{b} \times n_{b}$ pencil $A_{b}-\lambda E_{b}$ contains the $n_{b}$ poles of $G(\lambda)$ in $\Gamma_{b}$ and

$$
\operatorname{rank}\left[\begin{array}{ll}
E_{b} & E_{b g}
\end{array}\right]=n_{b},
$$

that is, all infinite nondynamic modes are included in $A_{g}-\lambda E_{g}$. Starting with an arbitrary minimal realization (6) it is always possible to arrive to a separated realization by determining first a realization as in (15), and further making a spectral decomposition of the pencil $A_{d}-\lambda E_{d}$ with respect to the partition (1). Furthermore, if the realization to start with has real coefficients, and $\Gamma_{g}$ is symmetric, we can always determine a separated realization with real coefficients as well.

\section{Basic Pole Displacement Result}

In this section we solve the following general pole displacement problem (PDP).

PDP: Given a $p \times m$ rational matrix $G$ and a disjoint partition $\overline{\mathbb{C}}=\Gamma_{g} \cup \Gamma_{b}$, both arbitrary, determine an invertible $p \times p$ rational matrix $M$ with all poles in $\Gamma_{g}$ which cancels by left multiplication all poles of $G$ located in $\Gamma_{b}$, i.e., such that $N(\lambda):=M(\lambda) G(\lambda)$ has all poles in $\Gamma_{g}$.

The following theorem gives an answer to the PDP in terms of descriptor realizations of $G$ and $M$. It is a rich extension of Theorem 3.1 and Lemma 4.1 in [17].

Theorem 3.1 Given a rational matrix $G(\lambda)$ and a disjoint partition $\overline{\mathbb{C}}=\Gamma_{g} \cup \Gamma_{b}$, both arbitrary, let $n_{b}$ be the number of poles of $G$ in $\Gamma_{b}$. Assume (19) is a minimal descriptor realization of $G$ separated with respect to the given partition and satisfying the condition (20). Then the class of solutions to the PDP is given by

$$
M(\lambda)=\left[\begin{array}{c|c}
A_{x}-\lambda E_{x} & B_{x}(\alpha-\beta \lambda) \\
\hline C_{x} & D_{x}
\end{array}\right]
$$

where the realization (21) is minimal and satisfies: $A_{x}-\lambda E_{x}$ has all eigenvalues in $\Gamma_{g}, D_{x}$ is invertible, $\frac{\alpha}{\beta} \notin \Lambda\left(A_{b}-\lambda E_{b}\right)$, and

$$
\left[\begin{array}{cc}
A_{x}-\lambda E_{x} & B_{x}(\alpha-\beta \lambda) \\
C_{x} & D_{x}
\end{array}\right]\left[\begin{array}{c}
X \\
C_{b}
\end{array}\right]=\left[\begin{array}{c}
Y \\
O
\end{array}\right]\left(A_{b}-\lambda E_{b}\right)
$$

holds for certain injective matrices $X$ and $Y$.

The minimal McMillan degree of a solution to the PDP is $n_{b}$.

Proof. Before proceeding with the proof we make several preparations. Using conversion formulas as for getting from (15) to (16), we can put (19) in the form

$$
G(\lambda)=\left[\begin{array}{cc|c}
A_{b}-\lambda E_{b} & \widetilde{A}_{b g}-\lambda \widetilde{E}_{b g} & \widetilde{B}_{b}-\lambda \widetilde{F}_{b} \\
O & \widetilde{A}_{g}-\lambda \widetilde{E}_{g} & \widetilde{B}_{g}-\lambda \widetilde{F}_{g} \\
\hline C_{b} & \widetilde{C}_{g} & \widetilde{D}
\end{array}\right]
$$


where

$$
\operatorname{rank}\left[\begin{array}{ccc}
E_{b} & \widetilde{E}_{b g} & \widetilde{F}_{b}
\end{array}\right]=n_{b}
$$

and

$$
\Lambda\left(A_{b}-\lambda E_{b}\right) \cap \Lambda\left(\widetilde{A}_{g}-\lambda \widetilde{E}_{g}\right)=\emptyset .
$$

This conversion is necessary as to enforce (25), and can be skipped if $\infty \in \Gamma_{g}$. Using (23) and (21) we can write a realization of $N(\lambda)=M(\lambda) G(\lambda)$ as

$$
N(\lambda)=\left[\begin{array}{ccc|c}
A_{x}-\lambda E_{x} & B_{x} C_{b}(\alpha-\beta \lambda) & B_{x} \widetilde{C}_{g}(\alpha-\beta \lambda) & B_{x} \widetilde{D}(\alpha-\beta \lambda) \\
O & A_{b}-\lambda E_{b} & \widetilde{A}_{b g}-\lambda \widetilde{E}_{b g} & \widetilde{B}_{b}-\lambda \widetilde{F}_{b} \\
O & O & \widetilde{A}_{g}-\lambda \widetilde{E}_{g} & \widetilde{B}_{g}-\lambda \widetilde{F}_{g} \\
\hline C_{x} & D_{x} C_{b} & D_{x} \widetilde{C}_{g} & D_{x} \widetilde{D}
\end{array}\right] .
$$

Assume $X$ and $Y$ are two matrices that satisfy the generalized Sylvester equation

$$
\left(A_{x}-\lambda E_{x}\right) X-Y\left(A_{b}-\lambda E_{b}\right)+B_{x} C_{b}(\alpha-\beta \lambda)=0
$$

and let $\widetilde{X}$ and $\widetilde{Y}$ be the unique solutions that satisfy the generalized Sylvester equation

$$
\left(A_{b}-\lambda E_{b}\right) \tilde{X}-\tilde{Y}\left(\widetilde{A}_{g}-\lambda \widetilde{E}_{g}\right)+\widetilde{A}_{b g}-\lambda \widetilde{E}_{b g}=0 .
$$

Note that $\tilde{X}$ and $\tilde{Y}$ always exist due to (25). Define

$$
Q:=\left[\begin{array}{ccc}
I & -Y & O \\
O & I & -\widetilde{Y} \\
O & O & I
\end{array}\right], \quad Z:=\left[\begin{array}{ccc}
I & X & O \\
O & I & \widetilde{X} \\
O & O & I
\end{array}\right]
$$

By using the transformation matrices (29) we obtain an equivalent realization in the form

$$
N(\lambda)=\left[\begin{array}{ccc|c}
A_{x}-\lambda E_{x} & O & * & * \\
O & A_{b}-\lambda E_{b} & O & \widehat{B}_{b}-\lambda \widehat{F}_{b} \\
O & O & \widetilde{A}_{g}-\lambda \widetilde{E}_{g} & \widetilde{B}_{g}-\lambda \widetilde{F}_{g} \\
\hline C_{x} & D_{x} C_{b}+C_{x} X & * & D_{x} \tilde{D}
\end{array}\right],
$$

where from the minimality of $G(\lambda)$ and from (24) we have

$$
\begin{gathered}
\operatorname{rank}\left[\begin{array}{cc}
A_{b}-\lambda E_{b} & \widehat{B}_{b}-\lambda \widehat{F}_{b}
\end{array}\right]=n_{b}, \quad \forall \lambda \in \mathbb{C}, \\
\operatorname{rank}\left[\begin{array}{cc}
E_{b} & \widehat{F}_{b}
\end{array}\right]=n_{b} .
\end{gathered}
$$

We show first that if $M(\lambda)$ is given by the minimal realization (21) satisfying the respective conditions in the statement then it is a solution to the PDP. Notice first that $M(\lambda)$ is invertible. Since $\Lambda\left(A_{x}-\lambda E_{x}\right) \subset \Gamma_{g}$ we get that all poles of $M(\lambda)$ are in $\Gamma_{g}$. From (22) it will follow that $N(\lambda)=M(\lambda) G(\lambda)$ has all its poles in $\Gamma_{g}$. Indeed, (22) shows that (27) is satisfied and let $\tilde{X}$ and $\tilde{Y}$ be the unique solutions to (28). We perform an equivalence transformation with $Q$ and $Z$ defined by (29) to get to (30). From (22) we also get that the entry $D_{x} C_{b}+C_{x} X$ in (30) is zero. Thus, after removing the unobservable part we get

$$
N(\lambda):=\left[\begin{array}{cc|c}
A_{x}-\lambda E_{\mathrm{x}} & * & * \\
O & \widetilde{A}_{g}-\lambda \widetilde{E}_{g} & \widetilde{B}_{g}-\lambda \widetilde{F}_{g} \\
\hline C_{x} & * & D_{x} \widetilde{D}
\end{array}\right]
$$

which clearly has all its poles in $\Gamma_{g}$. Thus $M(\lambda)$ has canceled by left multiplication all poles of $G(\lambda)$ located in $\Gamma_{b}$ and thus it is a solution to the PDP.

Conversely, let now $M(\lambda)$ be a solution to the PDP and let (21) be a minimal realization of it, with $D_{x}$ invertible. From minimality it follows that $\Lambda\left(A_{x}-\lambda E_{x}\right) \subset \Gamma_{g}$. Hence $\Lambda\left(A_{x}-\lambda E_{x}\right) \cap$ 
$\Lambda\left(A_{b}-\lambda E_{b}\right)=\emptyset$ and thus there exist unique matrices $X$ and $Y$ to satisfy the generalized Sylvester equation (27). For similar reasons, the equation (28) has unique solutions $\widetilde{X}$ and $\widetilde{Y}$. Define $Q$ and $Z$ as in (29) and perform an equivalence transformation to get (30). Since $M(\lambda)$ is a solution to the PDP it follows that $N(\lambda)$ has no poles in $\Gamma_{b}$. However, (30) shows that this is possible only if

$$
\left(D_{x} C_{b}+C_{x} X\right)\left(A_{b}-\lambda E_{b}\right)^{-1}\left(\widehat{B}_{b}-\lambda \widehat{F}_{b}\right)
$$

has no poles in $\Gamma_{b}$. Since (31) holds, it follows by standard minimality arguments that (33) has no poles in $\Gamma_{b}$ only if

$$
D_{x} C_{b}+C_{x} X=0 \text {. }
$$

Combining this with (27) we obtain

$$
\left[\begin{array}{cc}
A_{x}-\lambda E_{x} & B_{x}(\alpha-\beta \lambda) \\
C_{x} & D_{x}
\end{array}\right]\left[\begin{array}{c}
X \\
C_{b}
\end{array}\right]=\left[\begin{array}{l}
Y \\
O
\end{array}\right]\left(A_{b}-\lambda E_{b}\right)
$$

It only remains to show that $X$ and $Y$ are injective. Since $M(\lambda)$ is invertible, the left-most pencil in (34) is regular. Thus, there exists $\lambda=\lambda_{0}$ for which the right-most and left-most pencils in (34) are invertible from where it follows that

$$
\operatorname{rank}\left[\begin{array}{c}
X \\
C_{b}
\end{array}\right]=\operatorname{rank}\left[\begin{array}{l}
Y \\
O
\end{array}\right]
$$

We show that $X$ is injective and the injectiveness of $Y$ follows then from (35). Let $\mathcal{V}=\operatorname{Ker} X$. Since $D_{x}$ is invertible, we get from (34) that

$$
C_{b} \mathcal{V}=0 \text {. }
$$

From (34) we get further

$$
\left[\begin{array}{l}
Y \\
O
\end{array}\right]\left(A_{b}-\lambda E_{b}\right) \mathcal{V}=0
$$

from where

$$
A_{b} \mathcal{V}+E_{b} \mathcal{V} \subset \operatorname{Ker} Y
$$

But (35) and (36) show that

$$
\operatorname{dim} \mathcal{V}=\operatorname{dim} \operatorname{Ker}\left[\begin{array}{l}
X \\
C_{b}
\end{array}\right]=\operatorname{dim} \operatorname{Ker}\left[\begin{array}{l}
Y \\
O
\end{array}\right]=\operatorname{dim} \operatorname{Ker} Y
$$

Thus

$$
\operatorname{dim}\left(A_{b} \mathcal{V}+E_{b} \mathcal{V}\right) \leq \operatorname{dim} \operatorname{Ker} Y=\operatorname{dim} \mathcal{V}
$$

where only equality can take place, since, in general, for a regular pencil $A_{b}-\lambda E_{b}$ and an arbitrary space $\mathcal{V}$ we have $\operatorname{dim}\left(A_{b} \mathcal{V}+E_{b} \mathcal{V}\right) \geq \operatorname{dim} \mathcal{V}$. It follows that

$$
\operatorname{dim}\left(A_{b} \mathcal{V}+E_{b} \mathcal{V}\right)=\operatorname{dim} \mathcal{V}
$$

and thus $\mathcal{V}$ is a deflating subspace (see [16]) of the pencil $A_{b}-\lambda E_{b}$ contained in $\operatorname{Ker} C_{b}$. However, this is possible only if $\mathcal{V}=0$ [15] because (23) is a minimal realization of $G(\lambda)$ and thus

$$
\operatorname{rank}\left[\begin{array}{c}
A_{b}-\lambda E_{b} \\
C_{b}
\end{array}\right]=n_{b} \quad \forall \lambda \in \mathbb{C}, \quad \operatorname{rank}\left[\begin{array}{c}
E_{b} \\
C_{b}
\end{array}\right]=n_{b} .
$$

It follows that $X$ and $Y$ are both injective and thus any solution to the PDP satisfies the requirements in the statement.

Finally, form the injectiveness of $X$ it follows that $\delta(M) \geq n_{b}$. 
Remark 3.2 It is easy to see that by an appropriate equivalence transformation, each solution $M(\lambda)$ of the PDP has a minimal realization (21) such that

$$
\left[\begin{array}{cc|c}
A_{x, 11}-\lambda E_{x, 11} & A_{x, 12}-\lambda E_{x, 12} & B_{x, 1}(\alpha-\beta \lambda) \\
A_{x, 21}-\lambda E_{x, 21} & A_{x, 22}-\lambda E_{x, 22} & B_{x, 2}(\alpha-\beta \lambda) \\
\hline C_{x, 1} & C_{x, 2} & D_{x}
\end{array}\right]\left[\begin{array}{c}
I \\
O \\
C_{b}
\end{array}\right]=\left[\begin{array}{c}
I \\
O \\
O
\end{array}\right]\left(A_{b}-\lambda E_{b}\right),
$$

where (40) follows directly from (22). Then it can be rapidiy checked that $M^{-1}(\lambda)$ has a realization of the form

$$
M^{-1}(\lambda)=\left[\begin{array}{cc|c}
A_{b}-\lambda E_{b} & * & * \\
O & * & * \\
\hline C_{b} & * & *
\end{array}\right]
$$

that is, $M^{-1}(\lambda)$ and $G(\lambda)$ have a "common" pair $\left(C_{b}, A_{b}-\lambda E_{b}\right)$. This fact shows the connections of our result with the "spectral triples" discussed in [8] for an invertible and proper $G(\lambda)$, aud will be instrumental in obtaining solutions to the noncanonical problems in Sections 5 and 6.

\section{Minimal Degree Coprime Factorization}

For a given rational matrix $G(\lambda)$ and a domain $\Gamma_{g}$, both arbitrary, we characterize now the class of LCFs over $\Gamma_{g}$ having minimal degree which we show to be $n_{b}$. Recall that $n_{b}$ is the number of poles of $G(\lambda)$ in $\Gamma_{b}$. For our proofs, we need the following result on the solution iu a particular form to the generalized eigenvalue assignment problem.

Lemma 4.1 Let $A-\lambda E$ be a regular pencil, with $A, E \in \mathbb{F}^{n \times n}, B \in \mathbb{F}^{n \times m}$, let $\Gamma \subset \overline{\mathbb{C}}$ be a set of $n$ elements (not necessarily distinct, and assumed to be symmetric if $\mathbb{F}=\mathbb{R}$ ), and let $\alpha, \beta \in \mathbb{F}$, not both zero, such that $\frac{\alpha}{\beta} \notin \Lambda(A-\lambda E)$ and $\frac{\alpha}{\beta} \notin \Gamma$. Assume that

$$
\begin{aligned}
& \operatorname{rank}\left[\begin{array}{ll}
A-\lambda E & B
\end{array}\right]=n, \quad \forall \lambda \in \mathbb{C}, \\
& \operatorname{rank}\left[\begin{array}{ll}
E & B
\end{array}\right]=n .
\end{aligned}
$$

Then there exists a matrix $F \in \mathbb{F}^{m \times n}$ such that

$$
\Lambda(A-\lambda E+B F(\alpha-\beta \lambda))=\Gamma
$$

Proof. To reduce (43) to a standard eigenvalue assignment problem we use the conformal mapping $\lambda=(\alpha z+\beta) /(\beta z-\alpha)$. Under this mapping $\Gamma$ changes to $\Gamma_{z}$, which will contain only finite points. Define $A_{z}-z E_{z}:=\alpha A+\beta E-z(\beta A-\alpha E)$ and $B_{z}:=\left(\alpha^{2}+\beta^{2}\right) B$. With the transformed data we have reduced $(43)$ to

$$
\Lambda\left(A_{z}+B_{z} F-z E_{z}\right)=\Gamma_{z}
$$

with $E_{z}$ invertible. From assumptions (i), for $z \neq \frac{\alpha}{\beta}$, and (ii), for $z=\frac{\alpha}{\beta}$, we get

$$
\operatorname{rank}\left[A_{z}-z E_{z} B_{z}\right]=n, \quad \forall z \in \mathbb{C}
$$

Thus (44) is a standard eigenvalue assignment problem for the controllable pair $\left(E_{z}^{-1} A_{z}, E_{z}^{-1} B_{z}\right)$ and the set $\Gamma_{z}$, and has always a solution $F$ which is the solution of the original problem (43) as well. If $\mathbb{F}=\mathbb{R}$, and $\Gamma$ is symmetric, then $\Gamma_{z}$ will be symmetric as well, and $F$ can be chosen with elements in $\mathbb{R}$.

Theorem 4.2 Given a rational matrix $G(\lambda)$ and a disjoint partition $\overline{\mathbb{C}}=\Gamma_{g} \cup \Gamma_{b}$, both arbitrary, let $n_{b}$ be the number of poles of $G$ in $\Gamma_{b}$. Assume (19) is a minimal descriptor realization of $G$ separated with respect to the given partition and satisfying the condition (20). Then we have:

1. The minimal degree of a $L C F$ of $G$ over $\Gamma_{g}$ is $n_{b}$. 
2. The class of solutions to the minimal degree LCF problem $G(\lambda)=M^{-1}(\lambda) N(\lambda)$ is given by

$$
\begin{gathered}
N(\lambda)=\left[\begin{array}{cc|c}
A_{b}-\lambda E_{b}+K C_{b}(\alpha-\lambda \beta) & A_{b g}-\lambda E_{b g}+K C_{g}(\alpha-\lambda \beta) & B_{b}+K D(\alpha-\lambda \beta) \\
O & A_{g}-\lambda E_{g} & B_{g} \\
\hline W C_{g} & W D
\end{array},\right. \\
\hline W C_{b} \\
M(\lambda)=\left[\begin{array}{c|c}
A_{b}-\lambda E_{b}+K C_{b}(\alpha-\lambda \beta) & K(\alpha-\lambda \beta) \\
\hline W C_{b} & W
\end{array}\right]
\end{gathered}
$$

where $W$ is an arbitrary invertible matrix, $K$ is any matrix that solves the generalized eigenvalue assignment problem $\Lambda\left(A_{b}-\lambda E_{b}+K C_{b}(\alpha-\lambda \beta)\right) \subset \Gamma_{g}$, and $\alpha$ and $\beta$ are chosen to satisfy the requirements of Lemma 4.1 .

If $G$ has real coefficients and $\Gamma_{g}$ is a symmetric set then the class of solutions with real coefficients to the minimal degree LCF problem is also given by (45) and (46) where now all the intervening elements can be chosen real.

Proof. We show first that $N(\lambda)$ and $M(\lambda)$ of the form (45)-(46) solve the minimal LCF problem with degree $n_{b}$. To show that $G(\lambda)=M^{-1}(\lambda) N(\lambda)$ is a matter of straightforward computations involving realizations and equivalence transformations. Further, we see from $(46)$ that $\delta(M(\lambda)) \leq n_{b}$. But we have also from Theorem 3.1 that $\delta(M(\lambda)) \geq n_{b}$ since $M(\lambda)$ solves in particular the corresponding PDP formulated for $G(\lambda)$. Thus $\delta(M(\lambda))=n_{b}$ which proves part 1 of the Theorem. We show further that $M(\lambda)$ and $N(\lambda)$ are left coprime. As both $N(\lambda)$ and $M(\lambda)$ have poles only in $\Gamma_{g}$, it is enough to show that the compound rational matrix $[N(\lambda) M(\lambda)]$ has no zeros outside $\Gamma_{g}$ (see [14] and [24] for equivalent characterizations of left coprimeness). Indeed, using the descriptor realization of $G=(A-\lambda E, B, C, D)$ separated as in (19), a realization of $[N(\lambda) M(\lambda)]$ is

$$
[N(\lambda) M(\lambda)]=\left[\begin{array}{c|cc}
A-\lambda E+\bar{K} C(\alpha-\lambda \beta) & B+\bar{K} D(\alpha-\lambda \beta) \bar{K}(\alpha-\lambda \beta) \\
\hline W C & W D
\end{array}\right]
$$

where

$$
\bar{K}=\left[\begin{array}{l}
K \\
O
\end{array}\right] .
$$

Incidentally, it is easy to see that (47) is a minimal realization. From Theorem 2.1 we have that the zeros of $[N(\lambda) M(\lambda)]$ are among the zeros (including infinity) of the system pencil associated with the above realization and given by

$$
\left[\begin{array}{ccc}
A-\lambda E+\bar{K} C(\alpha-\lambda \beta) & B+\bar{K} D(\alpha-\lambda \beta) & \bar{K}(\alpha-\lambda \beta) \\
W C & W D & W
\end{array}\right]
$$

which is strictly equivalent to (see [6] for the definition of strict equivalence for matrix pencils)

$$
\left[\begin{array}{ccc}
A-\lambda E & B & \bar{K}(\alpha-\lambda \beta) \\
O & O & W
\end{array}\right]
$$

Thus the zeros of $[N(\lambda) M(\lambda)]$ are among those of $[A-\lambda E B]$. However, this last pencil has no zeros since the representation of $G(\lambda)$ is minimal, and thus $\operatorname{rank}[A-\lambda E B]=n \forall \lambda \in \mathbb{C}, \lambda$ finite, and $\operatorname{rank}[E B]=n$. We conclude that $[N(\lambda) M(\lambda)]$ has no zeros as well and therefore $M(\lambda)$ and $N(\lambda)$ result left coprime over $\Gamma_{g}$. Actually, $M(\lambda)$ and $N(\lambda)$ result left coprime (over the whole $\overline{\mathbb{C}}$ ).

Conversely, we show now that if $M(\lambda)$ and $N(\lambda)$ solve the LCF over $\Gamma_{g}$ with minimal degree $n_{b}$ then they are of the form (45)-(46). Since $M(\lambda)$ and $N(\lambda)$ have all poles in $\Gamma_{g}$, it follows from Theorem 3.1 that there exists a minimal realization (21) of $M(\lambda)$ which satisfies (22). Since the McMillan degree of $M(\lambda)$ is precisely $n_{b}$, the injective matrices $X$ and $Y$ in $(22)$ are actually square and thus nonsingular. Therefore, using Remark 3.2, we can simply take $X=Y=-I_{n_{b}}$. Then, the expression for $M(\lambda)$ follows immediately from (22), where $B_{x}:=K$ solves the eigenvalue assignment problem $\Lambda\left(A_{b}-\lambda E_{b}+K C_{b}(\alpha-\lambda \beta)\right) \subset \Gamma_{g}$. Lemma 4.1 shows that this last problem always has a solution since from the minimality of the realization (19) we get that the pair $\left(A_{b}^{*}-\lambda E_{b}^{*}, C_{b}^{*}\right)$ satisfies 
(42) written for actual data, and $\alpha$ and $\beta$ can always be a priori chosen to satisfy the respective requirements. The expression (45) results immediately after a simple equivalence transformation and removing the unobservable part from the descriptor representation of the product $N(\lambda)=$ $M(\lambda) G(\lambda)$.

For the case of real coefficients, we can start with a separated realization for $G$ having real elements. Then we choose $\alpha$ and $\beta$ real, and since $\Gamma_{g}$ is symmetric it follows form Lemma 4.1 that the feedback matrix $K$ can be chosen with real elements as well.

Notice from the above theorem that the class of all solutions depends essentially only on the pair $\left(C_{b}, A_{b}-\lambda E_{b}\right)$ that is unique (up to an equivalence transformation) for a given $G(\lambda)$.

\section{LCF with $J$ all-pass denominator with respect to the imag- inary axis}

In this section we solve the minimal degree LCF with the additional requirement on the denominator to have a certain symmetry, namely we consider here the case in which the denominator is $J$ all-pass with respect to the imaginary axis. To reflect this symmetry accordingly, we take throughout this section the disjoint partition $\overline{\mathbb{C}}=\Gamma_{g} \cup \Gamma_{b}$ defined by

$$
\Gamma_{b}:=\mathbb{C}^{+}\left(\text {or } \Gamma_{b}:=\mathbb{C}^{-}\right) \quad \Gamma_{g}:=\overline{\mathbb{C}} \backslash \Gamma_{b} .
$$

However, due to the additional requirement on the denominator to be $J$ all-pass it is not always possible to solve the LCF over $\Gamma_{g}$ with minimal degree $n_{b}$. When this is possible, we call the factorization canonical, otherwise we call it noncanonical. Notice that for the both choices in (49), $\Gamma_{b}$ is an open set while $\Gamma_{g}$ is a closed one. This will be instrumental for the solution in the noncanonical case.

The following result that will prove useful later gives a characterization of proper $J$ all-pass rational matrices in terms of minimal realizations. It is a particular case (with $\alpha=1, \beta=0$ ) of the general Theorem A.1 given in Appendix.

Corollary 5.1 Let $M(s)$ be a proper square invertible rational matrix, having a minimal realization

$$
M(s):=\left[\begin{array}{c|c}
A-s E & B \\
\hline C & D
\end{array}\right] .
$$

Then $M$ is $J$ all-pass with respect to the imaginary axis if and only if

$$
D^{*} J D=J
$$

and there exists an invertible Hermitic matrix $X$ such that

$$
\begin{gathered}
A^{*} X E+E^{*} X A-C^{*} J C=0, \\
C-D J B^{*} X E=0 .
\end{gathered}
$$

\subsection{The canonical case}

The following theorem gives a complete solution to the LCF problem with $J$ all-pass denominator of McMillan degree $n_{b}$.

Theorem 5.2 Given an arbitrary rational matrix $G(s)$ and a disjoint partition $\overline{\mathbb{C}}=\Gamma_{g} \cup \Gamma_{b}$ defined by (49), tet $n_{b}$ be the number of poles of $G$ in $\Gamma_{b}$. Assume (19) is a minimal descriptor realization of $G$ separated with respect to the given partition and satisfying the condition (20). Then the $L C F$ with $J$ all-pass denominator with respect to the imaginary axis has a solution $G(s)=M^{-1}(s) N(s)$ of minimal degree $n_{b}$ if and only if the equation

$$
A_{b}^{*} X E_{b}+E_{b}^{*} X A_{b}-C_{b}^{*} J C_{b}=0
$$


has an invertible Hermitic solution $X$. In this case, the class of all solutions is given by

$$
\begin{gathered}
N(s)=\left[\begin{array}{cc|c}
A_{b}+K C_{b}-s E_{b} & A_{b g}+K C_{g}-s E_{b g} & B_{b}+K D \\
O & A_{g}-s E_{g} & B_{g} \\
\hline W C_{g} & W D
\end{array}\right], \\
M(s)=\left[\begin{array}{c|c}
A_{b}+K C_{b}-s E_{b} & K \\
\hline W C_{b} & W
\end{array}\right],
\end{gathered}
$$

where

$$
K=-X^{-1} E_{b}^{-*} C_{b}^{*} J
$$

and $W$ is any $J$ unitary matrix satisfying

$$
W^{*} J W=J .
$$

If $G$ has real coefficients then the class of solutions with real coefficients is also given by (55) and (56) where now all the intervening coefficients can be chosen real.

Proof. Notice first that $E_{b}$ in a minimal realization (19) is invertible due to the choice of $\Gamma_{b}$ in (49).

We show first that any solution $G=M^{-1} N$ to the LCF with $J$ all-pass denominator of McMillan degree $n_{b}$ is given by (55) and (56). In particular, $M$ and $N$ solve the minimal degree LCF problem (without any requirement on $M$ ) and must have minimal realizations of the form (45) and (46), respectively, where we can take $\alpha=1$ and $\beta=0$. Thus $N$ and $M$ are of the form (55) and (56), respectively. Since the realization (56) is minimal, and $M(s)$ is $J$ all-pass, it follows from Corollary 5.1 that (54) has an invertible Hermitic solution and (57) and (58) are satisfied. Moreover, it follows that $K$ given by (57) is such that $\Lambda\left(A_{b}+K C_{b}-s E_{b}\right) \subset \Gamma_{g}$.

Conversely, we have to show that $N$ and $M$ given by (55) and (56) are a solution to the LCF with $J$ all-pass denominator of McMillan degree $n_{b}$. But this follows immediately from Theorem 4.2 and Corollary 5.1.

For the case of real coefficients, we can start with a separated realization for $G$ having real elements, and it will follow from (54) that $X$ is real and symmetric.

If in the statement of the above theorem we add the condition $X>0$ or $X<0$ we obtain the solution to the LCF with $J$ lossless or $J$ expansive denominator, respectively (see [10]). Notice that for the chosen partition (49) the equation (54) has always a unique solution, and thus the above theorem is an effective tool for checking the existence of and computing the solutions to the LCF with $J$ all-pass denominator or, as a particular case, with $J$ lossless denominator. We do not elaborate further in this direction.

For $J=I$, the above theorem provides the solution of an important particular case: LCF with all-pass denominator $M(s)$, (i.e., $M^{*}(-\bar{s}) M(s)=I$ ). In this case, the equation (54) has always a sign definite (positive or negative) solution. If we take $\Gamma_{b}=\mathbb{C}^{+}$then we obtain the well known results for the LCF with inner denominator.

\subsection{The noncanonical case}

We study now LCFs with $J$ all-pass denominator in the noncanonical case, i.e., when there is no solution with a $J$ all-pass denominator of minimal McMillan degree $n_{b}$. This case is considerably more intricate than the canonical case. The idea is to introduce additional poles and zeros in $M(s)$ such that it could simultaneously be $J$ all-pass and solve the LCF problem for $G(s)$. At the same time, we want to keep the McMillan degree of $M$ as small as possible. It turns out that the additional poles/zeros can be taken only on the imaginary axis (including infinity) since the pole-zero symmetry featured by a $J$ all-pass factor implies with necessity that all additional poles will be reflected into symmetric additional zeros that will be also zeros of the compound matrix $[N(s) M(s)]$.

Before stating our main result we construct a particular separated realization of $G$ which facilitates the subsequent developments. Let $G(s)$ be an arbitrary rational matrix given by a minimal 
realization (19) separated with respect to $\Gamma_{g} \cup \Gamma_{b}$ and satisfying (20), and let $n_{b}$ be the number of poles of $G$ in $\Gamma_{b}$. Then the generalized Lyapunov equation

$$
A_{b}^{*} X E_{b}+E_{b}^{*} X A_{b}-C_{b}^{*} J C_{b}=0
$$

has a unique Hermitic solution $X$, and let $r:=\operatorname{rank}(X)$. Let

$$
U^{*} X U=\left[\begin{array}{cc}
\Sigma_{r} & O \\
O & O
\end{array}\right]
$$

be a spectral decomposition of $X$ where $\Sigma_{r}$ is diagonal and nonsingular and $U$ is unitary. Further, let

$$
U^{*} E_{b} Q^{*}=\left[\begin{array}{cc}
E_{11} & O \\
E_{21} & E_{22}
\end{array}\right]
$$

be a RQ decomposition of $U^{*} E_{b}$, where $Q$ is unitary, $E_{11}$ and $E_{22}$ are lower triangular, square and invertible, and the partition in (61) corresponds to the partition in (60). With (60) and (61) it is easy to see that

$$
G(s)=\left[\begin{array}{cc|c}
U^{*}\left(A_{b}-\lambda E_{b}\right) Q^{*} & U^{*}\left(A_{b g}-\lambda E_{b g}\right) & U^{*} B_{b} \\
O & A_{g}-\lambda E_{g} & B_{g} \\
\hline C_{b} Q^{*} & C_{g} & D
\end{array}\right]
$$

is another realization of $G$ separated with respect to $\Gamma_{g} \cup \Gamma_{b}$ which we call balanced with respect to the Lyapunov equation (59). Thus we may assume from the beginning that for the separated realization (19) we have $E_{b}$ lower triangular and the corresponding Lyapunov equation (59) has a diagonal solution. We are now ready for our main result.

Theorem 5.3 Given an arbitrary rational matrix $G(s)$ and a disjoint partition $\overline{\mathbb{C}}=\Gamma_{g} \cup \Gamma_{b}$ defined by (49), let $n_{b}$ be the number of poles of $G$ in $\Gamma_{b}$. Assume (19) is a minimal descriptor realization of $G$, separated with respect to the given partition, and satisfying the condition (2O). Let $r:=\operatorname{rank}(X)$ where $X$ is the unique Hermitic solution to the Lyapunov equation

$$
A_{b}^{*} X E_{b}+E_{b}^{*} X A_{b}-C_{b}^{*} J C_{b}=0
$$

Then the minimal degree of the $L C F$ with $J$ all-pass denominator is

$$
2 n_{b}-r \text {. }
$$

Proof. Without restricting the generality, we may assume from the beginning that the realization (19) is balanced with respect to the Lyapunov equation (63). Further, as $E_{b}$ is invertible we could, as a first simplifying step in the whole proof, reduce the equation (63) to a standard Lyapunov equation with $E_{b}=I$. However, we prefer not to invert $E_{b}$ as far as possible as this will bring benefits in terms of the reliability of the associated numerical algorithms.

The proof is quite lengthy and we divide it in several steps: we show first that any solution $G=M^{-1} N$ to the LCF with $J$ all pass denominator satisfies

$$
\delta(M) \geq 2 n_{b}-r,
$$

and prove further that $M^{-1}$ has exactly $n_{b}$ poles in $\Gamma_{b}$ (which are the poles of $G$ in $\Gamma_{b}$ ) and the rest (additional) $\delta(M)-n_{b}$ poles are with necessity on $\overline{\mathbb{C}}^{0}$. Finally, we construct a solution of minimal degree $2 n_{b}-r$ with the additional $n_{b}-r$ poles placed arbitrary on $\overline{\mathbb{C}}^{0}$.

Proof of $\delta(M) \geq 2 n_{b}-r$. Let $G(s)=M^{-1}(s) N(s)$ be a solution to the LCF with $J$ all pass denominator. As $M$ cancels in the product $N=M G$ all poles of $G$ in $\Gamma_{b}$ and $M^{-1}$ is $J$ all-pass, we get with Remark 3.2 and Theorem A.1 in Appendix that there exists a minimal realization of $M^{-1}$ of the form

$$
M^{-1}(s)=\left[\begin{array}{c|c}
A_{M}-s E_{M} & B_{M}(\alpha-s \beta) \\
\hline C_{M} & D_{M}
\end{array}\right]=\left[\begin{array}{cc|c}
A_{b}-s E_{b} & A_{b x}-s E_{b x} & B_{x 1}(\alpha-s \beta) \\
O & A_{x}-s E_{x} & B_{x 2}(\alpha-s \beta) \\
\hline C_{b} & C_{x} & D_{M}
\end{array}\right]
$$


and there exists a Hermitic invertible matrix $X_{M}$ such that

$$
A_{M}^{*} X_{M} E_{M}+E_{M}^{*} X_{M} A_{M}-C_{M}^{*} J C_{M}=0 .
$$

According to the partition of the right-hand side of $(66), X$ can be written in the partitioned form

$$
X_{M}=\left[\begin{array}{cc}
X & X_{12} \\
X_{12}^{*} & X_{22}
\end{array}\right]
$$

where it follows that $X$ is the unique solution to $(63)$. Since $X_{M}$ is invertible and the rank of $X$ is $r$, it follows from (68) that $\operatorname{rank}\left(X_{M}\right) \geq 2 n_{b}-r$ and thus (65) holds.

Location of the poles of $M^{-1}$. Let $G=M^{-1} N$ be a solution to the LCF with $J$ all pass denominator. From (66) we see that the union of poles of $M^{-1}(s)$ contains

$$
\Lambda\left(A_{b}-s E_{b}\right) \subset \mathbb{C}^{+}
$$

which are also the poles of $G$ in $\Gamma_{b}$. From the pole-zero symmetry of a $J$ all-pass rational matrix it follows that the poles of $M(s)$ are the conjugated of the poles of $M^{-1}(s)$. Since $M$ has all poles in $\Gamma_{g}$ we infer that the poles of $M^{-1}$ are in $\bar{\Gamma}_{b}:=\Gamma_{b} \cup \overline{\mathbb{C}}^{0}$ from where we have

$$
\Lambda\left(A_{x}-s E_{x}\right) \subset \bar{\Gamma}_{b} \text {. }
$$

We show now that

$$
\Lambda\left(A_{x}-s E_{x}\right) \subset \Gamma_{g}
$$

from where we shall conclude with (70) that

$$
\Lambda\left(A_{x}-s E_{x}\right) \subset \overrightarrow{\mathbb{C}}^{0}
$$

and thus $M^{-1}$ has exactly $n_{b}$ poles in $\Gamma_{b}$ and the additional $\delta(M)-n_{b}$ poles are on $\overline{\mathbb{C}}^{0}$. We prove in fact that the coprimeness of $N$ and $M$ over $\Gamma_{g}$ implies (71). To this end we compute realizations for $M$ and $N$. Using (66) we write a realization of $M$ in the form

$$
\begin{aligned}
M(s) & =\left[\begin{array}{c|c}
A_{M}-s E_{M}-B_{M} W C_{M}(\alpha-s \beta) & -B_{M} W(\alpha-s \beta) \\
\hline W C_{M} & W
\end{array}\right] \\
& =\left[\begin{array}{cc|c}
A_{b}-s E_{b}-B_{x 1} W C_{b} p(s) & A_{b x}-s E_{b x}-B_{x 1} W C_{x} p(s) & -B_{x 1} W p(s) \\
-B_{x 22} W C_{b} p(s) & A_{x}-s E_{x}-B_{x 2} W C_{x} p(s) & -B_{x 2} W p(s) \\
\hline W C_{b}
\end{array}\right],
\end{aligned}
$$

where $W:=D_{M}^{-1}$ and $p(s):=\alpha-s \beta$. With (73) and (19) we get successively a realization of

$$
N(s)=M(s) G(s)
$$

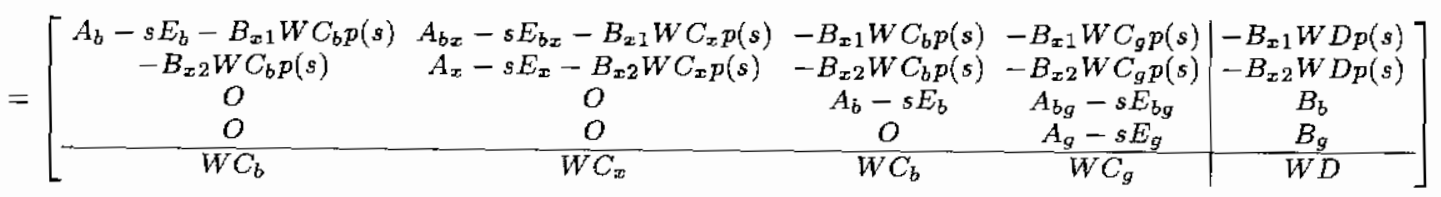

$$
=\left[\begin{array}{ccc|c}
A_{b}-s E_{b}-B_{x 1} W C_{b} p(s) & A_{b x}-s E_{b x}-B_{x 1} W C_{x} p(s) & A_{b g}-B_{x 1} W C_{g} p(s)-s E_{b g} & B_{b}-B_{x 1} W D_{p}(s) \\
-B_{x 2} W C_{b} p(s) & A_{x}-s E_{x}-B_{x 2} C_{x} p(s) & -B_{x 2} W C_{g} p(s) & -B_{x 2} W D p(s) \\
O & 0 & A_{g}-s E_{g} & B_{g} \\
\hline W C_{b} & W & W C_{g}
\end{array}\right]
$$

where we have removed the unobservable part with the help of a state-space equivalence transformation. From (74) we see that the poles of $N(s)$ are among $\Lambda\left(A_{M}-s E_{M}-B_{M} W C_{M}(\alpha-s \beta)\right) \cup$ $\Lambda\left(A_{g}-s E_{g}\right)$ and thus they are indeed included in $\Gamma_{g}$. We use now (73) and (74) to write the system pencil associated with the compound matrix $[N(s) M(s)]$ in the form

$\left[\begin{array}{ccccc}A_{b}-s E_{b}-B_{x 1} W C_{b} p(s) & A_{b x}-s E_{b x}-B_{x 1} W C_{x} p(s) & A_{b g}-B_{x 1} W C_{g} p(s)-s E_{b g} & B_{b}-B_{x 1} W D_{p}(s) & -B_{x 1} W p(s) \\ -B_{x 2} W C_{b} p(s) & A_{x}-s E_{x}-B_{x 2} C_{x} p(s) & -B_{x 2} W C_{g} p(s) & -B_{x 2} W D p(s) & -B_{x 2} W p(s) \\ O & O & A_{g}-s E_{g} & O \\ W C_{b} & W C_{x} & W C_{g} & W D & W\end{array}\right]$. 
The zeros of $[N(s) M(s)]$ are among the zeros of the above pencil which is strictly equivalent to

$$
\left[\begin{array}{ccccc}
A_{b}-s E_{b} & A_{b g}-s E_{b g} & B_{b} & A_{b x}-s E_{b x} & -B_{x 1} W p(s) \\
O & A_{g}-s E_{g} & B_{g} & O & O \\
O & O & O & A_{x}-s E_{x} & -B_{x 2} W p(s) \\
O & O & O & O & W
\end{array}\right] .
$$

The realization (19) of $G$ is minimal and thus controllable, for where it follows that the only zeros of $[N(s) M(s)]$ are given by $\Lambda\left(A_{x}-s E_{x}\right)$ which should be included in $\Gamma_{g}$ since $N$ and $M$ are coprime over $\Gamma_{g}$. Thus we proved that the coprimeness of $N$ and $M$ over $\Gamma_{g}$ implies that (71) holds.

Construction of a minimal solution. We show now that the LCF with $J$ all-pass denominator has a solution $G=M^{-1} N$ such that

$$
\delta(M) \leq 2 n_{b}-r
$$

from where it will follow with (65) that the minimal degree $2 n_{b}-r$ can be achieved. To this end, we shall construct $M(s)$, define $N(s)=M(s) G(s)$, and prove that they both satisfy the required properties. Of course, $M$ should simultaneously be $J$ all-pass, and $M^{-1}$ should have the form (66), with the additional poles given by $\Lambda\left(A_{x}-s E_{x}\right) \subset \overline{\mathbb{C}}^{0}$.

For the sake of clarity we assume first that the additional poles/zeros $\Lambda\left(A_{x}-s E_{x}\right)$ are placed in finite locations on $\mathbb{C}^{0}$. The case with poles at infinity follows analogously although the formulas become more intricate.

Placing additional poles/zeros in finite locations on $\mathbb{C}^{0}$. In this case we can take for $M^{-1}$ a realization (66) with $\alpha=1, \beta=0$, i.e.,

$$
M^{-1}(s)=\left[\begin{array}{c|c}
A_{M}-s E_{M} & B_{M} \\
\hline C_{M} & D_{M}
\end{array}\right]=\left[\begin{array}{cc|c}
A_{b}-s E_{b} & A_{b x}-s E_{b x} & B_{x 1} \\
O & A_{x}-s E_{x} & B_{x 2} \\
\hline C_{b} & C_{x} & D_{M}
\end{array}\right] .
$$

The key idea of the construction is to embed the Lyapunov equation (63) into a larger Lyapunov equation (67) with an invertible solution $X_{M}$ as in (68) and where the matrices $A_{x}, E_{x}, A_{b x}, E_{b x}$, $B_{x 1}, B_{x 2}, C_{x}$ have to be determined. We can simply take

$$
E_{b x}=0, \quad E_{x}=I_{n_{b}-r}
$$

in case of placing additional poles/zeros at finite locations. We choose further

$$
X_{12}=\left[\begin{array}{c}
O \\
I_{n_{b}-r}
\end{array}\right], \quad X_{22}=O
$$

and get

$$
\begin{array}{cc}
X_{M}=\left[\begin{array}{ccc}
\Sigma_{r} & O & O \\
O & O & I_{n_{b}-r} \\
O & I_{n_{b}-r} & O
\end{array}\right], & E_{M}=\left[\begin{array}{ccc}
E_{11} & O & O \\
E_{21} & E_{22} & O \\
O & O & I_{n_{b}-r}
\end{array}\right], \\
A_{M}=\left[\begin{array}{ccc}
A_{11} & A_{12} & A_{x 1} \\
A_{21} & A_{22} & A_{x 2} \\
O & O & A_{x}
\end{array}\right], \quad C_{M}=\left[\begin{array}{lll}
C_{1} & C_{2} & C_{x}
\end{array}\right],
\end{array}
$$

where we have taken into account that the realization (19) is balanced with respect to (63) and we have partitioned

$$
A_{b}=\left[\begin{array}{ll}
A_{11} & A_{12} \\
A_{21} & A_{22}
\end{array}\right], \quad A_{b x}=\left[\begin{array}{l}
A_{x 1} \\
A_{x 2}
\end{array}\right], \quad C_{b}=\left[\begin{array}{ll}
C_{1} & C_{2}
\end{array}\right],
$$

conformably with (60). The matrices $A_{x 1}, A_{x 2}, A_{x}, C_{x}$ remain to be determined as to satisfy (67). We use (80) to write (67) component-wise as

$$
A_{11}^{*} \Sigma_{\mathrm{r}} E_{11}+E_{11}^{*} \Sigma_{r} A_{11}-C_{1}^{*} J C_{1}=0
$$




$$
\begin{gathered}
E_{11}^{*} \Sigma_{r} A_{12}-C_{1}^{*} J C_{2}=0 \\
C_{2}^{*} J C_{2}=0 \\
A_{21}^{*}+E_{11}^{*} \Sigma_{\tau} A_{x 1}+E_{21}^{*} A_{x}-C_{1}^{*} J C_{x}=0 \\
A_{22}^{*}+E_{22}^{*} A_{x}-C_{2}^{*} J C_{x}=0 \\
A_{x 2}^{*}+A_{x 2}-C_{x}^{*} J C_{x}=0
\end{gathered}
$$

where the rest of equations are transpose conjugated versions of the ones above. Equations (81), (82) and (83) are automatically fulfilled due to (63). Before showing how the remaining equations can be satisfied, we prove that the pair $\left(C_{2}, A_{22}-s E_{22}\right)$ is observable. Indeed, since the pair $\left(C_{\bar{b}}, A_{b}-s E_{b}\right)$ is observable, the standard PHB observability tests shows that

$$
\operatorname{rank}\left[\begin{array}{c}
A_{b}-s E_{b} \\
C_{b}
\end{array}\right]=\operatorname{rank}\left[\begin{array}{cc}
A_{11}-s E_{11} & \Sigma_{r}^{-1} E_{11}^{-*} C_{1}^{*} J C_{2} \\
A_{21}-s E_{22} & A_{22}-s E_{22} \\
C_{1} & C_{2}
\end{array}\right]=n_{b}, \quad \forall s \in \mathbb{C}
$$

where we have replaced $A_{12}=\Sigma_{r}^{-1} E_{11}^{-*} C_{1}^{*} J C_{2}$ from (82). From (87) we get

$$
\operatorname{rank}\left[\begin{array}{c}
A_{22}-s E_{22} \\
C_{2}
\end{array}\right]=n_{b}-r, \quad \forall s \in \mathbb{C} .
$$

Thus $\left(C_{2}, A_{22}-s E_{22}\right)$ is observable. It follows from (85) that we can take the matrix $C_{x}$ such that the poles of $A_{x}=E_{22}^{-*}\left(-A_{22}^{*}+C_{2}^{*} J C_{x}\right)$ are on the imaginary axis by simply solving an eigenvalue assignment problem for the controllable pair

$$
\left(-E_{22}^{-*} A_{22}^{*}, E_{22}^{-*} C_{2}^{*} J\right)
$$

Alternatively, we may compute $C_{x}$ as to solve a generalized eigenvalue assignment problem for the controllable pair $\left(-A_{22}^{*}+s E_{22}^{*},-C_{2}^{*} J\right)$ with the benefit of not inverting $E_{22}^{*}$. Finally, we choose $A_{x 1}$ as the unique solution of (84) and $A_{x 2}$ as any solution to (86). With all these choices it results that (67) is satisfied.

We take $D_{M}$ to be any $J$ unitary matrix and define

$$
B_{M}=\left[\begin{array}{c}
B_{x 1} \\
B_{x 2}
\end{array}\right]:=E_{M}^{-*} X_{M}^{-1} C_{M}^{*} D_{M}^{*} J
$$

We get from Corollary 5.1 that $M^{-1}$ given in (78) is $J$ all pass. It follows that $M$ has all its poles in $\Gamma_{g}$, is $J$ all-pass, has McMillan degree less or equal to $2 n_{b}-r$, and a realization is given by

$$
\begin{aligned}
M(s) & =\left[\begin{array}{c|c|c}
A_{M}-s E_{M}-B_{M} W C_{M} & -B_{M} W \\
\hline W C_{M} & W
\end{array}\right] \\
& =\left[\begin{array}{cc|c}
A_{\bar{b}}-s E_{b}-B_{x 1} W C_{b} p(s) & A_{b x}-B_{x 1} W C_{x} p & -B_{x 1} W \\
\hdashline-B_{x 2} W C_{b} & A_{x}-s I-B_{x 2} W C_{x} & -B_{x 2} W \\
\hline W C_{b} & W C_{x} & W
\end{array}\right]
\end{aligned}
$$

where $W=D_{M}^{-1}$. We define $N(s):=M(s) G(s)$ and get

$$
N(s)=\left[\begin{array}{ccc|c}
A_{b}-s E_{b}-B_{x 1} W C_{b} & A_{b x}-B_{x 1} W C_{x} & A_{b g}-B_{x 1} W C_{g}-s E_{b g} & B_{b}-B_{x 1} W D \\
-B_{x 2} W C_{b} & A_{x}-s I-B_{x 2} C_{x} & -B_{x 2} W C_{g} & -B_{x 2} W D \\
O & O & A_{g}-s E_{g} & B_{g} \\
\hline W \bar{C} C_{b} & W C_{x} & W C_{g} & W D
\end{array}\right] .
$$

We can check successively that $N$ has all poles in $\Gamma_{g}$, and that $N$ and $M$ are coprime over $\Gamma_{g}$. The details are skipped since the argument is identical as in the first part of the proof for showing that the additional poles of $M^{-1}$ should be placed on $\overline{\mathbb{C}}^{0}$. Thus $N$ and $M$ define a solution to the LCF 
with $J$ all-pass denominator, having degree less or equal to $2 n_{b}-r$. This together with (65) shows that the minimal degree $2 n_{b}-r$ is attained and concludes the proof.

Placing additional poles/zeros arbitrary on $\overline{\mathbb{C}^{0}}$. We show that this case can be reduced to the case of finite poles/zeros. Indeed, we chose a realization for $M(s)$ of the form (66), where we take $\alpha \in \mathbb{R}$ and $\beta \in \mathbb{C}^{0}$ such that $\frac{\alpha}{\beta}$ is neither an eigenvalue of $A_{b}-s E_{b}$ nor a desired location of additional poles/zeros. A characterization of $J$ all-pass rational matrices with a realization centered at $\frac{\alpha}{\beta}$ is given in Theorem A.1 in Appendix. Thus we have again to embed a Lyapunov equation (63) into a larger Lyapunov equation (67) but where now $E_{M}$ will be singular if we would like to place additional poles/zeros at infinity. To this end we transform the Lyapunov equation (67) into the equivalent Lyapunov equation

$$
\widehat{A}_{M}^{*} X_{M} \widehat{E}_{M}+\widehat{E}_{M}^{*} X_{M} \widehat{A}_{M}-\widehat{C}_{M}^{*} J \widehat{C}_{M}=0
$$

where the coefficients to be determined are

$$
\widehat{A}_{M}:=\alpha A_{M}+\beta E_{M}, \quad \widehat{E}_{M}:=\alpha E_{M}-\beta A_{M}, \quad \widehat{C}_{M}=\sqrt{\alpha^{2}+\beta^{2}} C_{M},
$$

with $\widehat{E}_{M}$ invertible. Thus we apply exactly the same procedure as for finite poles/zeros but now we determine the coefficients of the equation (92) instead of (67). Finally, the coefficients of (67) follow immediately from (93). The only precaution is with respect to the locations in which the additional poles/zeros have to be placed which are mapped by a bilinear transformations moving $\infty$ into the finite point $\frac{\alpha}{\beta} \in \mathbb{C}^{0}$. The rest of the argument is identical and is therefore not repeated.

The proof of Theorem 5.3 gives as well a procedure to compute solutions of minimal McMillan degree. Moreover, the result below shows that from (78) one can get the class of all solutions.

Theorem 5.4 Assume the same notation and hypotheses as for Theorem 5.3. The class of all solutions to the minimal degree $L C F$ with $J$ all-pass denominator $G=M^{-1} N$, with $M$ proper, is given by (90) and (91), where $C_{x}$ and $A_{x}$ follow by solving the eigenvalue assignment problem for the pair (88) with $A_{x}=E_{22}^{-*}\left(-A_{22}^{*}+C_{2}^{*} J C_{x}\right) \subset \mathbb{C}^{0}, A_{x 2}$ is any solution to (86), $A_{x 1}$ is the unique solution to (84), $W$ is any $J$ unitary matrix, and $B_{x 1}$ and $B_{x 2}$ are given by (89).

Proof. Most of the proof is already contained in the proof of Theorem 5.3. We have only to prove that any solution may be obtained by the procedure given in the statement. Let $G=M^{-1} N$ be a solution to the LCF with $J$ all pass denominator $M$ of minimal degree $2 n_{b}-r$, and proper. Let $G$ be given by a separated realization (19) balanced with respect to the Lyapunoy equation (63). Then $M^{-1}$ is given by a realization (78), and (67) is satisfied for an invertible Hermitic matrix $X_{M}$ as in (68), where $X$ has the form in the right-hand side of $(60)$. We show that by equivalence state-space transformations on (78) we obtain a realization for which the corresponding Lyapunov equation (67) has all the intervening matrices of the form depicted in (80), and this will end the proof.

Writing $X_{M}$ in partitioned form we get

$$
X_{M}=\left[\begin{array}{ccc}
\Sigma_{r} & O & X_{1} \\
O & O & X_{2} \\
X_{1}^{*} & X_{2}^{*} & X_{22}
\end{array}\right]
$$

where we have denoted $X_{12}=\left[\begin{array}{c}X_{1} \\ X_{2}\end{array}\right]$. Since the matrix $X_{M}$ is square and invertible, and has dimension $2 n_{b}-r$ we get that $X_{2}$ is invertible, and thus we can write

$$
U_{M}^{*} X_{M} U_{M}=\left[\begin{array}{ccc}
\Sigma_{r} & O & O \\
O & O & I_{n_{b}-r} \\
O & I_{n_{b}-r} & O
\end{array}\right], U_{M}:=\left[\begin{array}{ccc}
I & O & -\Sigma_{\tau} X_{1} X_{2}^{-1} \\
O & I & -\frac{1}{2} X_{2}^{-*}\left(X_{22}-X_{1}^{*} \Sigma_{\tau}^{-1} X_{1}\right) X_{2}^{-1} \\
O & O & X_{2}^{-1}
\end{array}\right]
$$

Further, let

$$
\widetilde{E}_{M}:=U_{M}^{-1} E_{M}=\left[\begin{array}{cc}
\widetilde{E}_{b} & \widetilde{E}_{b x} \\
O & \widetilde{E}_{\mathfrak{x}}
\end{array}\right]
$$


where the block upper triangular structure in the right-hand side follows from the block upper triangular structure of both $E_{M}$ and $U_{M}^{-1}$. With $\widehat{E}_{M}:=I, \widehat{A}_{M}:=U_{M}^{-1} A_{M} \widetilde{E}_{M}^{-1}, \widehat{C}_{M}:=C_{M} \widetilde{E}_{M}^{-1}$ and $\hat{X}_{M}:=U_{M}^{*} X_{M} U_{M}$ we get that $(67)$ can be written equivalently as

$$
\widehat{A}_{M}^{*} \widehat{X}_{M} \widehat{E}_{M}+\widehat{E}_{M}^{*} \widehat{X}_{M} \widehat{A}_{M}-\widehat{C}_{M}^{*} J \widehat{C}_{M}=0
$$

where the intervening matrices with "hat" have the same block structure as their counterparts in $(80)$. It follows that the corresponding equations $(81)-(86)$ are satisfied from where the conclusion is immediate.

The class of all solutions, including the case of improper $M$, is obtained from (73) and (74) where the unknown coefficients are obtained by using first the transformation (93) to reduce the problem to the case of a proper $M$. We do not elaborate further in this direction.

Remark 5.5 From the proof of Theorem 5.3 we can give an answer to the general problem of existence of a LCF with $J$ lossless denominator as follows. If the solution $X$ to the equation (63) is positive definite, then the problem is solvable, otherwise there is no solution of any degree. Indeed, assume by contrary that $X$ is not positive definite and there is a solution to the LCF with $J$ lossless denominator of a certain degree. Then the equation (67) should be satisfied with $X_{M}>0$ given in (68), from where $X>0$ which contradicts our assumption.

\section{LCF with $J$ all-pass denominators with respect to the unit circle}

In this section we give the discrete-time version of the results presented in Section 6. More precisely, we solve the minimal degree LCF problem with the additional requirement on the denominator to have another type of symmetry, namely to be $J$ all-pass with respect to the unit circle. Throughout this section we take the disjoint partition $\overline{\mathbb{C}}=\Gamma_{g} \cup \Gamma_{b}$ defined by

$$
\Gamma_{b}:=\mathbb{D}^{c}\left(\text { or } \Gamma_{b}:=\mathbb{D}\right), \quad \Gamma_{g}:=\overline{\mathbb{C}} \backslash \Gamma_{b} .
$$

Similarly as for the symmetry discussed in the previous section, it is not always possible to solve the LCF over $\Gamma_{g}$ with minimal degree $n_{b}$. Again, when this is possible, we call the factorization canonical, otherwise we call it noncanonical.

Since our theory encompasses the cases in which $M(z)$ has to cancel a pole of $G$ at $\infty$ or at 0 , it follows that $M(z)$ or $M^{-1}(z)$ could, in general, be improper. Therefore, to achieve the full generality we have to use for $M(z)$ a descriptor representation of type (9), with singular $E$. The following result gives a characterization of $J$ all-pass rational matrices (possible improper) without poles at 1 in terms of associated realizations. It is a particular case for $\alpha=\beta=1$ of the general Theorem A.2 given in Appendix.

Corollary 6.1 Let $M(z)$ be a square invertible rational matrix, without poles at 1 , having a minimal realization

$$
M(z):=\left[\begin{array}{c|c}
A-z E & (1-z) B \\
\hline C & D
\end{array}\right] .
$$

Then $M$ is $J$ all-pass with respect to the unit circle if and only if

$$
D^{*} J D=J
$$

and there exists an invertible Hermitic matrix $X$ such that

$$
\begin{gathered}
E^{*} X E-A^{*} X A-C^{*} J C=0, \\
C-D J B^{*} X(E-A)=0 .
\end{gathered}
$$




\subsection{The canonical case}

The following theorem gives a complete solution to the LCF with $J$ all-pass denominator of McMillan degree $n_{b}$.

Theorem 6.2 Given an arbitrary rational matrix $G(z)$ and a disjoint partition $\overline{\mathbb{C}}=\Gamma_{g} \cup \Gamma_{b}$ defined by (96), let $n_{b}$ be the number of poles of $G$ in $\Gamma_{b}$. Assume (19) is a minimal descriptor realization of $G$ separated with respect to the given partition and satisfying the condition (20). Then the LCF with $J$ all-pass denominator with respect to the unit circle has a solution $G(z)=M^{-1}(z) N(z)$ of minimal degree $n_{b}$ if and only if the Stein equation

$$
E_{b}^{*} X E_{b}-A_{b}^{*} X A_{b}-C_{b}^{*} J C_{b}=0
$$

has an invertible Hermitic solution $X$. In this case the class of all solutions is given by

$$
\begin{gathered}
N(z)=\left[\begin{array}{cc|c}
A_{b}-z E_{b}+K C_{b}(1-z) & A_{b g}-z E_{b g}+K C_{g}(1-z) & B_{b}+K D(1-z) \\
O & A_{g}-z E_{g} & B_{g} \\
\hline W C_{g} & W D
\end{array}\right] \\
M(z)=\left[\begin{array}{l|c}
A_{b}-z E_{b}+K C_{b}(1-z) & K(1-z) \\
\hline W C_{b} & W
\end{array}\right]
\end{gathered}
$$

where

$$
K=-X^{-1}\left(E_{b}-A_{b}\right)^{-*} C_{b}^{*} J
$$

and $W$ is any $J$ unitary matrix satisfying

$$
W^{*} J W=J .
$$

If $G$ has real coefficients then the class of solutions with real coefficients is also given by (102) and (103) where now all the intervening coefficients can be chosen real.

Proof. The proof is identical as for Theorem 5.2 where we replace Corollary 5.1 with Corollary 6.1 .

If in the statement of the above theorem we add the condition $X>0$ or $X<0$ we obtain the solution to the LCF with $J$ lossless or $J$ expansive denominator, respectively. Notice that for the chosen partition (96) the equation (101) has always a unique solution, and thus the above theorem is an effective tool for checking the existence of and for computing solutions to the LCF with $J$ all-pass denominator or, as a particular case, with $J$ lossless denominator.

For $J=I$, the above theorem provides the solution of an important particular case: LCF with all-pass denominator $M(z)$, (i.e., $M^{*}(1 / \bar{z}) M(z)=I$ ). In this case, the equation (101) has always a sign definite (positive or negative) solution. If we take $\Gamma_{b}=\mathbb{D}^{\mathrm{c}}$ then we obtain the LCF with inner denominator.

\subsection{The noncanonical case}

We give now solvability conditions in the noncanonical case.

Theorem 6.3 Given an arbitrary rational matrix $G(z)$ and a disjoint partition $\overline{\mathbb{C}}=\Gamma_{g} \cup \Gamma_{b}$ defined by (96), let $n_{b}$ be the number of poles of $G$ in $\Gamma_{b}$. Assume (19) is a minimal descriptor realization of $G$, separated with respect to the given partition, and satisfying the condition (20). Let $r:=\operatorname{rank}(X)$ where $X$ is the unique Hermitic solution to the Stein equation (101). Then the minimal degree of the $L C F$ with $J$ all-pass denominator is

$$
2 n_{b}-r
$$


Proof. The proof is almost identical to the proof of Theorem 5.3. The key is to transform first the equation (101) into the equivalent equation

$$
\widehat{A}_{b}^{*} X \widehat{E}_{b}+\widehat{E}_{b}^{*} X \widehat{A}_{b}-\widehat{C}_{b}^{*} J \widehat{C}_{b}=0,
$$

where

$$
\widehat{A}_{b}:=E_{b}+A_{b}, \quad \widehat{E}_{b}:=\bar{E}_{b}-A_{b}, \quad \widehat{C}_{b}:=\sqrt{2} C_{b} .
$$

Since $\widehat{E}_{b}$ is invertible and (105) is of the form (63) we may use further a similar technique as in the proof of Theorem 5.3. We note that the role of $\infty$ in placing additional poles/zeros in the proof of Theorem 5.3 is taken in this case by the point 1 .

A remark similar to Remark 5.5 holds in this case as well.

\section{Numerical Aspects and Examples}

In this section we comment briefly on the numerical algorithms that are recommended for the methods proposed so far, and give several numerical examples. All the computations can be performed by procedures available in a recently developed DESCRIPTOR SYSTEMS Toolbox [21] implemented under MATLAB 5.1. This Toolbox uses extensively the object oriented approach for control systems analysis and design introduced within the CONTROL Toolbox 4.0. For all critical computations, the .mex files technology of MATLAB has been used, on basis of FORTRAN codes from LAPACK [1] and SLICOT [4] libraries.

\subsection{Numerical aspects}

Separated realizations as used in this paper can always be obtained by starting with an arbitrary realization (6) and performing unitary state-space equivalence transformations. If $\infty \in \Gamma_{b}$ then the preliminary transformation depicted in (15) is needed as to enforce further condition (20). The unitary matrices $Q$ and $Z$ in (15) can be obtained by a row compression of $E$ followed by a column compression of $A$. The row/column compressions can be achieved by using any rank revealing procedure [5] based on unitary transformations. Further, we can arrive at (19) satisfying (20) by performing a spectral decomposition of the regular pencil $A_{d}-\lambda E_{d}$ (or $A-\lambda E$ if the preliminary step is skipped). The spectral decomposition of a regular pencil can be achieved by unitary transformations using the QZ algorithm [11] followed by a reordering of the generalized eigenvalues [16].

For solving the generalized eigenvalue assignment problem in Lemma 4.1 we can adapt almost all existing algorithms for pole assignment but perhaps the most easy to customize is [18].

For solving generalized Lyapunov equations (63) or Stein equations (101) one can use the algorithms proposed in [7]. Once the solution computed, the balanced form can be obtained by solving a symmetric eigenyalue problem for $X[9]$. Again, we can rely solely on unitary transformations.

\subsection{Numerical examples}

\section{Example 1. Minimal degree factorization with proper factors}

Consider the improper rational matrix

$$
G(\lambda)=\left[\begin{array}{cc}
\lambda^{2} & \frac{\lambda}{\lambda \frac{1}{1}} \\
0 & \frac{1}{\lambda}
\end{array}\right]
$$


having finite poles at $\lambda_{1}=1$ and $\lambda_{2}=0$ and two poles at infinity, and let $\Gamma_{g}=\mathbb{C}^{-}$. A minimal order descriptor realization for $G$ in the form (19) is given by

$$
\begin{gathered}
{\left[\begin{array}{c|c}
A_{b}-\lambda E_{b} & A_{b g}-\lambda E_{b g} \\
\hline O & A_{g}-\lambda E_{g}
\end{array}\right]=\left[\begin{array}{cccc|c}
-\lambda & 1 & 0 & 0 & 0 \\
0 & 1-\lambda & 0 & 0 & 0 \\
0 & 0 & 1 & -\lambda & 0 \\
0 & 0 & 0 & 1 & -\lambda \\
\hline 0 & 0 & 0 & 0 & 1
\end{array}\right], \quad\left[\begin{array}{c}
B_{b} \\
\hline B_{g}
\end{array}\right]=\left[\begin{array}{rr}
0 & 0 \\
0 & -1 \\
0 & 0 \\
0 & 0 \\
\hline 1 & 0
\end{array}\right]} \\
{\left[C_{b} \mid C_{g}\right]=\left[\begin{array}{rrrr|r}
0 & -1 & -1 & 0 & 0 \\
1 & -1 & 0 & 0 & 0
\end{array}\right], \quad D=\left[\begin{array}{ll}
0 & 1 \\
0 & 0
\end{array}\right] .}
\end{gathered}
$$

To compute a minimal degree $\mathrm{LCF}$ over $\Gamma_{g}$ we use Theorem 4.2 and determine first a matrix $K$ such that

$$
\Lambda\left(A_{b}-\lambda E_{b}+K C_{b}(\alpha-\beta \lambda)\right)=\{-1,-1,-1,-1\},
$$

where we chose for convenience $\alpha=-2$ and $\beta=1$. By using the Lemma 4.1 for the dual pair $\left(A_{b}^{T}-\lambda E_{b}^{T}, C_{b}^{T}\right)$ we can assign the eigenvalues for the modified pair

$$
\left(A_{z}-\lambda E_{z}, C_{z}\right):=\left(\alpha A_{b}^{T}-\beta E_{b}^{T}-\lambda\left(\beta A_{b}^{T}-\alpha E_{b}^{T}\right),\left(\alpha^{2}+\beta^{2}\right) C_{b}^{T}\right)
$$

to $\Gamma_{z}:=\{3,3,3,3\}$, where $E_{z}$ is nonsingular. By usiug a pole assignment algorithm for generalized eigenvalues [18], we determined

$$
K=\left[\begin{array}{rr}
-8 & 1 \\
-8 & 0 \\
6 & 0 \\
3 & 0
\end{array}\right]
$$

which fulfills (106). Using (45) and (46), we get the following solution to the minimal degree LCF over $\Gamma_{g}$ :

$$
N(\lambda)=\left[\begin{array}{cc}
\frac{(\lambda-1) \lambda^{2}}{3(\lambda+1)^{3}} & \frac{\lambda}{3(\lambda+1)^{3}} \\
0 & \frac{1}{2(\lambda+1)}
\end{array}\right], \quad M(\lambda)=\left[\begin{array}{cc}
\frac{\lambda-1}{3(\lambda+1)^{3}} & 0 \\
0 & \frac{\lambda}{2(\lambda+1)}
\end{array}\right] .
$$

where the minimal degree of the LCF is clearly 4.

\section{Example 2. Minimal degree coprime factorization with $J$ all-pass denom- inator with respect to the imaginary axis}

Consider the TFM of a continuous-time system

$$
G(s)=\left[\begin{array}{ll}
\frac{1}{s \bar{\gamma}_{1}^{2}} & \frac{1}{s-1} \\
\frac{s-2}{s-1}
\end{array}\right]
$$

where $\gamma_{1}, \gamma_{2} \in \mathbb{R}$ are two parameters, and let $\Gamma_{b}=\mathbb{C}^{+}$. It is easy to check that $G$ has two unstable finite poles at $s_{1}=1$ and $s_{2}=2$. A minimal order realization for $G$ in the form (19) is given by

$$
A_{b}-s E_{b}=\left[\begin{array}{cc}
1-s & 0 \\
0 & 2-s
\end{array}\right], \quad B_{b}=\left[\begin{array}{ll}
1 & 0 \\
0 & 1
\end{array}\right], \quad C_{b}=\left[\begin{array}{cc}
1 & 1 \\
\gamma_{1} & \gamma_{2}
\end{array}\right], \quad D=\left[\begin{array}{ll}
0 & 0 \\
0 & 0
\end{array}\right] .
$$

With $J=\left[\begin{array}{rr}1 & 0 \\ 0 & -1\end{array}\right]$, the solution to the Lyapunov equation (54) in Theorem 5.2 is

$$
X=\left[\begin{array}{cc}
\frac{1-\gamma_{1}^{2}}{2} & \frac{1-\gamma_{1} \gamma_{2}}{3} \\
\frac{1-\gamma_{1} \gamma_{2}}{3} & \frac{1-\gamma_{2}^{2}}{4}
\end{array}\right],
$$

which for $\gamma_{1}\left(\gamma_{2}+3\right)-1-3 \gamma_{2} \neq 0$ is nonsingular, for $\gamma_{1}\left(\gamma_{2}+3\right)-1-3 \gamma_{2}=0$ and $\gamma_{2} \neq 1$ has rank equal to 1 and for $\gamma_{1}=\gamma_{2}=1$ has rank equal to 0 . 


\section{The canonical case}

Consider $\gamma_{1}=2$ and $\gamma_{2}=1$ for which the solution of (54) is

$$
X=\left[\begin{array}{rr}
-\frac{3}{2} & -\frac{1}{3} \\
-\frac{1}{3} & 0
\end{array}\right]
$$

and the corresponding $K$ in Theorem 5.2 is

$$
K=-X^{-1} C_{b}^{T} J=\left[\begin{array}{cc}
3 & -3 \\
-\frac{21}{2} & \frac{15}{2}
\end{array}\right] .
$$

The resulting factors $N(s)$ and $M(s)$ are

$$
N(s)=\frac{1}{2}\left[\begin{array}{cc}
\frac{2 s+11}{(s+1)(s+2)} & \frac{2}{(s+1)} \\
\frac{4 s+13}{(s+1)(s+2)} & \frac{2}{(s+1)}
\end{array}\right], \quad M(s)=\frac{1}{2}\left[\begin{array}{cc}
\frac{2 s^{2}-9 s-5}{(s+1)(s+2)} & \frac{9 s-3}{(s+1)(s+2)} \\
\frac{-9 s+3}{(s+1)(s+2)} & \frac{2 s^{2}+9 s-5}{(s+1)(s+2)}
\end{array}\right] .
$$

The noncanonical case: $\operatorname{rank}(X)=1$

Consider $\gamma_{1}=2$ and $\gamma_{2}=5$ for which the solution of (54) is

$$
X=\left[\begin{array}{rr}
-\frac{3}{2} & -3 \\
-3 & -6
\end{array}\right]
$$

and has only a nonzero eigenvalue at $-\frac{15}{2}$. With

$$
U=\frac{1}{\sqrt{5}}\left[\begin{array}{rr}
1 & -2 \\
2 & 1
\end{array}\right]
$$

we obtain

$$
U^{T} X U=\left[\begin{array}{rr}
-\frac{15}{2} & 0 \\
0 & 0
\end{array}\right]
$$

and the known matrices in (80) result with $Q=U^{T}$ as

$$
\begin{gathered}
U^{T} A U:=\left[\begin{array}{c|c}
A_{11} & A_{12} \\
\hline A_{21} & A_{22}
\end{array}\right]=\frac{1}{5}\left[\begin{array}{ll}
9 & 2 \\
2 & 6
\end{array}\right], \quad C U:=\left[\begin{array}{c|c}
C_{1} \mid & C_{2}
\end{array}\right]=\frac{1}{\sqrt{5}}\left[\begin{array}{rr}
3 & -1 \\
12 & 1
\end{array}\right], \\
U^{T} E U=\left[\begin{array}{cc}
E_{11} & O \\
E_{21} & E_{22}
\end{array}\right]=\left[\begin{array}{ll}
1 & 0 \\
0 & 1
\end{array}\right] .
\end{gathered}
$$

The matrix

$$
X_{M}=\left[\begin{array}{rrr}
-\frac{15}{2} & 0 & 0 \\
0 & 0 & 1 \\
0 & 1 & 0
\end{array}\right]
$$

fulfills the equation (67) for

$$
A_{x 1}=\frac{8}{15}, \quad A_{x 2}=\frac{18}{5}, \quad A_{x}=0, \quad C_{x}=\left[\begin{array}{c}
-\frac{6}{\sqrt{5}} \\
0
\end{array}\right],
$$


where we have chosen $C_{x}$ such that $A_{x}$ has a zero eigenvalue. The corresponding $B_{M}$ results as

$$
B_{M}=X_{M}^{-1} C_{M}^{T} J=\frac{1}{\sqrt{5}}\left[\begin{array}{rr}
-\frac{2}{5} & \frac{8}{5} \\
-6 & 0 \\
-1 & -1
\end{array}\right]
$$

With $M^{-1}$ defined by (78) we obtain finally

$$
\begin{gathered}
M(s)=\frac{1}{75}\left[\begin{array}{cc}
\frac{75 s^{3}+63 s^{2}-336 s-50}{s(s+2)(s+1)} & -\frac{2\left(81 s^{2}-168 s+25\right)}{s(s+2)(s+1)} \\
\frac{2\left(81 s^{2}+168 s+25\right)}{s(s+2)(s+1)} & \frac{75 s^{3}-63 s^{2}-336 s+50}{s(s+2)(s+1)}
\end{array}\right] \\
N(s):=M(s) G(s)=\frac{1}{25}\left[\begin{array}{cc}
\frac{25 s^{2}-62 s+50}{s(s+2)(s+1)} & \frac{25 s^{2}-199 s+50}{s(s+2)(s+1)} \\
\frac{2\left(25 s^{2}+31 s-25\right)}{s(s+2)(s+1)} & \frac{125 s^{2}+199 s-50}{s(s+2)(s+1)}
\end{array}\right]
\end{gathered}
$$

The noncanonical case: $\operatorname{rank}(X)=0$

Consider $\gamma_{1}=\gamma_{2}=1$ for which the solution of (54) is $X=0$. With $U=I$, we further have $A_{22}=A$ and $C_{2}=C$. The matrix $X_{M}$ has the form

$$
X_{M}=\left[\begin{array}{llll}
0 & 0 & 1 & 0 \\
0 & 0 & 0 & 1 \\
1 & 0 & 0 & 0 \\
0 & 1 & 0 & 0
\end{array}\right]
$$

and fulfills the equation (67) for

$$
A_{x 2}=\left[\begin{array}{ll}
0 & 0 \\
0 & 0
\end{array}\right], \quad A_{x}=\left[\begin{array}{ll}
-3 & 5 \\
-2 & 3
\end{array}\right], \quad C_{x}=\left[\begin{array}{rr}
-1 & \frac{5}{2} \\
1 & -\frac{5}{2}
\end{array}\right],
$$

where this time we have chosen $C_{x}$ such that $A_{x}$ has two eigenvalues on the imaginary axis at $\pm j$. The corresponding $B_{M}$ results as

$$
B_{M}=X_{M}^{-1} C_{M}^{T} J=\left[\begin{array}{rr}
-1 & -1 \\
5 & 5 \\
\frac{2}{2} & \frac{2}{1} \\
1 & -1
\end{array}\right] .
$$

With $M^{-1}$ defined by (78) we obtain eventually

$$
\begin{gathered}
M(s)=\frac{1}{2}\left[\begin{array}{cc}
\frac{2 s^{4}-3 s^{2}+5}{\left(s^{2}+1\right)(s+2)(s+1)} & \frac{-7 s^{2}+3}{\left(s^{2}+1\right)(s+2)(s+1)} \\
\frac{-7 s^{2}+3}{\left(s^{2}+1\right)(s+2)(s+1)} & \frac{2 s^{4}-3 s^{2}+5}{\left(s^{2}+1\right)(s+2)(s+1)}
\end{array}\right], \\
N(s):=M(s) G(s)=\left[\begin{array}{cc}
\frac{s-2}{s^{2}+1} & \frac{s-1}{s^{2}+1} \\
\frac{s-2}{s^{2}+1} & \frac{s-1}{s^{2}+1}
\end{array}\right]
\end{gathered}
$$




\section{Example 3. Minimal degree coprime factorization with $J$ all-pass denom- inator with respect to the unit circle}

Consider the TFM of a discrete-time system

$$
G(z)=\left[\begin{array}{cc}
z^{2} & \frac{1}{z-2} \\
0 & z
\end{array}\right]
$$

having an unstable finite pole at $z_{1}=2$ and three poles at infinity, and let $\Gamma_{b}:=\mathbb{D}^{c}$. A minimal order descriptor realization for $G$ in the form (19) is given by

$$
\begin{gathered}
{\left[\begin{array}{c|c}
A_{b}-z E_{b} & A_{b g}-z E_{b g} \\
\hline O & A_{g}-z E_{g}
\end{array}\right]=\left[\begin{array}{rrrr|rr}
2-z & 0 & 0 & 0 & 0 & 0 \\
0 & 1 & -z & 0 & 0 & 0 \\
0 & 0 & 1 & 0 & -z & 0 \\
0 & 0 & 0 & 1 & 0 & -z \\
\hline 0 & 0 & 0 & 0 & 1 & 0 \\
0 & 0 & 0 & 0 & 0 & 1
\end{array}\right],\left[\begin{array}{c}
B_{b} \\
\hline B_{g}
\end{array}\right]=\left[\begin{array}{ll}
0 & 1 \\
0 & 0 \\
0 & 0 \\
0 & 0 \\
\hline 1 & 0 \\
0 & 1
\end{array}\right]} \\
{\left[C_{b} \mid C_{g}\right]=\left[\begin{array}{rrrr|rr}
1 & -1 & 0 & 0 & 0 & 1 \\
0 & 0 & 0 & -1 & 0 & 0
\end{array}\right], \quad D=\left[\begin{array}{ll}
0 & 1 \\
0 & 0
\end{array}\right] .}
\end{gathered}
$$

The solution of (101) is

$$
X=\left[\begin{array}{cccc}
-1 / 3 & 1 / 2 & 1 / 4 & 0 \\
1 / 2 & -1 & 0 & 0 \\
1 / 4 & 0 & -1 & 0 \\
0 & 0 & 0 & 1
\end{array}\right]
$$

and the corresponding $K$ in Theorem 6.2 is

$$
K=-X^{-1}\left(E_{b}-A_{b}\right)^{-T} C_{b}^{T} J=\left[\begin{array}{rr}
-12 & 0 \\
-5 & 0 \\
-2 & 0 \\
0 & 1
\end{array}\right]
$$

The factors $N(z)$ and $M(z)$ resulting from Theorem 6.2 are

$$
N(z)=\left[\begin{array}{cc}
-\frac{z-2}{2 z-1} & -\frac{1}{z^{2}(2 z-1)} \\
0 & 1
\end{array}\right], \quad M(z)=\left[\begin{array}{cc}
-\frac{z-2}{z^{2}(2 z-1)} & 0 \\
0 & \frac{1}{z}
\end{array}\right] .
$$

\section{Conclusions}

We have presented a comprehensive theory of minimal degree coprime factorization of rational matrices over a given domain of the closed complex plane. To some extent, the theory supports the already existing algorithms to compute coprime factors by recursive pole dislocation techniques $[17,19,20]$ and, moreover, provides a systematic theoretical framework for them. Nevertheless, in view of the formulas given in this paper there is no need to stick on recursive methods unless specific computational reasons justify this.

Recursive techniques work for coprime factorizations with proper, polynomial, or stable factors. They are are also applicable in case of factorizations with inner or $J$ lossless denominators. Generally, the recursive techniques are applicable whenever the involved Lyapunov equations have sign definite solutions (positive or negative), and then these equations are solved only implicitly. However, the recursive techniques can not be employed in the more general cases discussed in this paper, not even 
in the canonical $J$ all-pass case. Essentially, the recursive techniques fail since a $J$ all-pass rational matrix can not be written in general as a product of elementary $J$ all-pass factors.

A particular feature of the results developed in this paper is that the methods are exactly tailored to the dimension of the problem to be solved avoiding unnecessary redundancy. As an example, the solution of a full order Riccati equation (order equal to the McMillan degree of the rational matrix to be factored) that is usually employed in such factorizations (see for example [10], [26]) is completely avoided. Instead, we solve a Lyapunov equation of lower dimension with the benefits of increased numerical accuracy and computational efficiency.

The approach taken in this paper puts ground also for extensions to LCF with denominators satisfying other symmetries, as for example with respect to the real line, or with respect to a certain contour in the complex plane. The theory presented here has been already applied as a preliminary step to the computation of the most general inner-outer, spectral, and $J$ lossless factorizations [12], [13], and it is a promising step towards computing the more general $J$ spectral factorizations, either canonical or noncanonical.

\section{References}

[1] E. Anderson, Z. Bai, J. Bishop, J. Demmel, J. Du Croz, A. Greenbaum, S. Hammarling, A. McKenney, S. Ostrouchov, and D. Sorensen. LAPACK User's Guide, Second Edition. SIAM, Philadelphia, 1995.

[2] J.A. Ball, J. Kim, L. Rodman, M. Verma, Minimal degree coprime factorization of rational matrix functions, Linear Algebra and Its Applications, 186:117-164, 1993.

[3] H. Bart, I. Gohberg, M.A. Kaashoek. Constructive Methods of Wiener-Hopf Factorization. Birkhäuser, Basel, OT 21, 1986.

[4] P. Benner, V. Mehrmann, V. Sima, S. Van Huffel, and A. Varga. SLICOT - a subroutine library in systems and control theory. In B. N. Datta, editor, Applied and Computational Control, Signals and Circuits, volume 1. Birkhäser, 1997.

[5] T. Chan. Rank revealing QR factorizations. Lin. Alg. \& Appl., 88/89:67-82, 1987.

[6] F. R. Gantmacher. The Theory of Matrices. Chelsea, New York, 1960.

[7] J. D. Gardiner, A. J. Laub, J. J. Amato, and C. B. Moler. Solution of the Sylvester matrix equation $A X B^{T}+C X D^{T}=E$. ACM Trans. Math. Software, 18:223-231, 1992.

[8] I. Gohberg, M.A. Kaashoek, A.C.M. Ran. Partial pole and zero displacement by cascade connection. SIAM J. Matrix Anal. Appl., 10(3):316-325, 1989.

[9] G.H. Golub, Ch.F, van Loan. Matrix Computations. John Hopkins University Press, Baltimore, 1989.

[10] H. Kimura. Chain-Scattering Approach to $H_{\infty}$-Control. Birkhäuser, Boston, 1996.

[11] C. B. Moler and G. W. Stewart. An algorithm for generalized matrix eigenvalue problem. SIAM J. Numer. Anal., 10:241-256, 1973.

[12] C. Oară and A. Varga. Inner-outer factorization of rational matrices: the general case. In Proceedings of the MTNS'98, Padova, 1998.

[13] C. Oară and A. Varga. J-lossless factorization of descriptor systems In Proceedings of the Symphosium on Descriptor Systems, Paderborn, 1998.

[14] H. H. Rosenbrock. State-Space and Multivariable Theory. Wiley, New York, 1970.

[15] P. Van Dooren. The generalized eigenstructure problem in linear systems theory. IEEE Trans. Autom. Control, 26:111-129, 1981.

[16] P. Van Dooren. A generalized eigenvalue approach for solving Riccati equations. SIAM J. Sci. Stat. Comput., 2:121-135, 1981.

[17] P. Van Dooren. Rational and polynomial matrix factorizations via recursive pole-zero cancellation. Lin. Alg. \& Appl, 137/138:663-697, 1990. 
[18] A. Varga. On stabilization of descriptor systems. Systems \& Control Letters, 24:133-138, 1995.

[19] A. Varga. Computation of coprime factorizations of rational matrices. Lin. Alg. \& Appl., 271:83-115, 1998.

[20] A. Varga and T. Katayama. Computation of J-inner-outer factorizations of rational matrices. Int. J. Robust and Nonlinear Control, 1997. (to appear).

[21] A. Varga, C. Oară. A descriptor system toolbox for MATLAB. In Proceedings of the Symphosium on Descriptor Systems, Paderborn, 1998.

[22] G. Verghese, B. Lévy, and T. Kailath. A generalized state-space for singular systems. IEEE Trans. Autom. Control, AC-26:811-831, 1981.

[23] G. Verghese, P. Van Dooren, and T. Kailath. Properties of the system matrix of a generalized state-space system. Int. J. Control, 30:235-243, 1979.

[24] M. Vidyasagar. Control System Synthesis: A Factorization Approach. The M1T Press, Cambridge, MA, 1985.

[25] X. Xin and H. Kimura. $\left(J, J^{\prime}\right)$ lossless factorization for descriptor systems. Lin. Alg. \& Appl., 205-206:1289-1318, 1994.

[26] X. Xin and H. Kimura. Singular $\left(J, J^{\prime}\right)$ lossless factorization for strictly proper functions. Int. J. Control, 59:1383-1400, 1994.

[27] K. Zhou, J.C. Doyle, and K. Glover. Robust and Optimal Control. Prentice Hall, New Jersey, 1996.

\section{A Appendix}

In this Appendix we give a characterization in terms of associated realizations for an arbitrary $J$ all-pass rational matrix, with respect to either the imaginary axis or the unit circle. In this context we see the importance of centered realizations of the type (9) for which a suitable choice of $\alpha$ and $\beta$ provides very handy formulas. A suitable choice means that $\alpha$ and $\beta$ are such that $\frac{\alpha}{\beta}$ is on the contour with respect to which the symmetry is defined (either the imaginary axis or the unit circle). This choice guarautees, for a given rational matrix $M$ with a minimal realization centered at $\frac{\alpha}{\beta}$, that its adjoint $M^{\sim}$ has a minimal realization centered at $\frac{\alpha}{\beta}$ which can be simply expressed in terms of the realization of $M$. This is especially important when working with TFMs of discrete-time systems as it avoids the intricacies related to the feature that $\infty$ is the symmetrized value of 0 .

We start with the case in which the symmetry is with respect to the imaginary axis.

Theorem A.1 Let $M(s)$ be a square and invertible rational matrix. Let $\alpha \in \mathbb{R}$ and $\beta \in \mathbb{C}^{0}$ such that $\frac{\alpha}{\beta}$ is not a pole of $M$ and assume

$$
M(s):=\left[\begin{array}{c|c}
A-s E & B(\alpha-\beta s) \\
\hline C & \bar{D}
\end{array}\right] .
$$

is a minimal realization. Then $M$ is $J$ all-pass with respect to the imaginary axis if and only if

$$
D^{*} J D=J
$$

and there exists an invertible Hermitic matrix $X$ such that

$$
\begin{gathered}
A^{*} X E+E^{*} X A-C^{*} J C=0, \\
C-D J B^{*} X(\alpha E-\beta A)=0 .
\end{gathered}
$$

Proof. Notice first that $M(s)$ having no poles at $\frac{\alpha}{\beta}$ implies that in a minimal realization (107) $\alpha E-\beta A$ is invertible.

Only if. Since $M(s)$ is $J$ all-pass, we have

$$
M^{*}(-\bar{s}) J M(s)=J
$$


for all $s=j \omega$ which are not poles of $M(s)$. In particular, for $s=\frac{\alpha}{\beta}$ we get (108). Hence $D$ is invertible. Further, (111) may be rewritten as

$$
M^{*}(-\bar{s}) J=J M^{-1}(s)
$$

or, equivalently, in terms of realizations

$$
\left[\begin{array}{c|c|c}
-A^{*}-s E^{*} & C^{*} J(\alpha-\beta s) \\
\hline-B^{*} & D^{*} J
\end{array}\right]=\left[\begin{array}{c|c}
A-\alpha B D^{-1} C-s\left(E-\beta B D^{-1} C\right) & B D^{-1}(\alpha-s \beta) \\
\hline-J D^{-1} C
\end{array}\right] .
$$

Since both realizations in (112) are minimal and they realize the same rational matrix, there exist unique invertible matrices $Q$ and $Z$ such that

$$
\begin{gathered}
Q\left(A-\alpha B D^{-1} C\right) Z=-A^{*}, \\
Q\left(E-\beta B D^{-1} C\right) Z=E^{*}, \\
Q B D^{-1}=C^{*} J \\
J D^{-1} C Z=B^{*} .
\end{gathered}
$$

It is easy to see that equations (113)-(116) hold also with $Q$ and $Z$ replaced by $Z^{-*}$ and $Q^{-*}$, respectively. Due to the uniqueness of $Q$ and $Z$ we get that

$$
Q=Z^{-*} \text {. }
$$

Thus substituting (117) in (113) and (114) we get

$$
\begin{gathered}
\left(A-\alpha B D^{-1} C\right) Z=-Z^{*} A^{*}, \\
\left(E-\beta B D^{-1} C\right) Z=Z^{*} E^{*} .
\end{gathered}
$$

Rewriting (116) we get

$$
C-D J B^{*} Z^{-1}=0 .
$$

Comparing (120) with (110) we define

$$
X:=Z^{-1}(\alpha E-\beta A)^{-1}
$$

that is clearly invertible. To show that $X$ is Hermitic, we subtract (118) multiplied by $\beta$ from (119) multiplied by $\alpha$ and get

$$
X^{-1}=(\alpha E-\beta A) Z=Z^{*}\left(\alpha E^{*}+\beta A^{*}\right)=X^{-*} .
$$

It only remains to show (109). We rewrite the left-hand side of (109) successively as

$$
\begin{gathered}
\frac{1}{\alpha^{2}+\beta^{2}}\left[\left(-\beta E^{*}+\alpha A^{*}\right) X(\alpha E-\beta A)+\left(\alpha E^{*}+\beta A^{*}\right) X(\alpha A+\beta E)\right]-C^{*} J C \\
\stackrel{(121)}{=} \frac{1}{\alpha^{2}+\beta^{2}}\left[\left(-\beta E^{*}+\alpha A^{*}\right) Z^{-1}+Z^{-*}(\alpha A+\beta E)\right]-C^{*} J C \\
\stackrel{(118)-(119)}{=} Z^{-*} B D^{-1} C-C^{*} J C \stackrel{(120)}{=} 0
\end{gathered}
$$

which ends the first part of the proof.

If. We show that if (108)-(110) hold for an invertible Hermitic matrix $X$ then $M(s)$ is $J$ all-pass. Starting with (107) we get a realization of

$$
M^{\sim}(s) J M(s)=\left[\begin{array}{cc|c}
-A^{*}-s E^{*} & C^{*} J C & C^{*} J D(\alpha-\beta s) \\
O & A-s E & B(\alpha-\beta s) \\
\hline-B^{*} & D^{*} J C & D^{*} J D
\end{array}\right] .
$$


Let

$$
Q:=\left[\begin{array}{cc}
I & -\left(\beta A^{*}+\alpha E^{*}\right) X \\
O & I
\end{array}\right], \quad Z:=\left[\begin{array}{cc}
I & X(\alpha E-\beta A) \\
O & I
\end{array}\right]
$$

and performing an equivalence state-space transformation with $Q$ to the left and $Z$ to the right we get

$$
\begin{aligned}
& M^{*}(-\bar{s}) J M(s) \\
& \quad=\left[\begin{array}{cc|c}
-A^{*}-s E^{*} & C^{*} J C-E^{*} X A-A^{*} X E & {\left[C^{*} J D-\left(\beta A^{*}+\alpha E^{*}\right) X B\right](\alpha-\beta s)} \\
\hline O & A-s E & B(\alpha-\beta s) \\
\hline-\overline{B^{*}} & -B^{*} X(\alpha E-\beta A)+D^{*} J C & D^{*} J D
\end{array}\right]
\end{aligned}
$$

Now using (108)-(110) we get that the $(1,2),(1,3)$ and $(3,2)$ entries of $(125)$ are identically zero. Thus removing the uncontrollable and unobservable part of (125) we get finally

$$
M^{\sim}(s) J M(s)=D^{*} J D
$$

from where the conclusion that $M(s)$ is $J$ all-pass.

Here follows the case of a $J$ all-pass rational matrix with respect to the unit circle.

Theorem A.2 Let $M(z)$ be a square and inverible rational matrix. Let $\alpha \in \mathbb{C}$, with $\|\alpha\|=1$ and such that $\alpha^{2}$ is not a pole of $M$, and assume

$$
M(z):=\left[\begin{array}{c|c}
A-z E & (\alpha-\bar{\alpha} z) B \\
\hline C & \bar{D}
\end{array}\right]
$$

is a minimal realization. Then $M$ is $J$ all-pass with respect to the unit circle if and only if

$$
D^{*} J D=J
$$

and there exists an invertible Hermitic matrix $X$ such that

$$
\begin{aligned}
& E^{*} X E-A^{*} X A-C^{*} J C=0, \\
& C-D J B^{*} X(\alpha E-\bar{\alpha} A)=0 .
\end{aligned}
$$

Proof. Notice first that since $M(z)$ has no pole at $\alpha^{2}$ and (126) is a minimal realization it follows that $\bar{\alpha} A-\alpha E$ is invertible.

Only if. Since $M(z)$ is $J$ all-pass, we have

$$
M^{\sim}(z) J M(z)=J
$$

for all $z=e^{j \theta}$ which are not poles of $M(z)$. In particular, for $z=\alpha$ we get (127) and it follows that $D$ is invertible. Further, (130) may be rewritten as

$$
M^{*}\left(\frac{1}{\bar{z}}\right) J=J M^{-1}(z)
$$

or, equivalently, in terms of realizations

$$
\left[\begin{array}{c|c|c}
E^{*}-z A^{*} & (\alpha-\bar{\alpha} z) C^{*} J \\
\hline B^{*} & D^{*} J
\end{array}\right]=\left[\begin{array}{c|c}
\left(A-\alpha B D^{-1} C\right)-z\left(E-\bar{\alpha} B D^{-1} C\right) & (\alpha-\bar{\alpha} z) B D^{-1} \\
\hline J D^{-1}
\end{array}\right] .
$$

Since both realizations in (132) are minimal and they realize the same rational matrix, there exists two unique invertible matrices $Q$ and $Z$ such that

$$
\begin{aligned}
& Q\left(A-\alpha B D^{-1} C\right) Z=E^{*}, \\
& Q\left(E-\bar{\alpha} B D^{-1} C\right) Z=A^{*},
\end{aligned}
$$




$$
\begin{gathered}
Q B D^{-1}=C^{*} J, \\
-J D^{-1} C Z=B^{*} .
\end{gathered}
$$

It is easy to see that equations (133)-(136) hold if we replace $Q$ and $Z$ with $-Z^{-*}$ and $-Q^{-*}$, respectively. Due to the uniqueness of $Q$ and $Z$ we get

$$
Q=-Z^{-*} \text {. }
$$

Thus substituting (137) in (133)-(136) results in

$$
\begin{aligned}
& \left(A-\alpha B D^{-1} C\right) Z=-Z^{*} E^{*}, \\
& \left(E-\bar{\alpha} B D^{-1} C\right) Z=-Z^{*} A^{*} .
\end{aligned}
$$

Rewriting (136) we get

$$
C+D J B^{*} Z^{-1}=0
$$

Comparing (129) with (140) we define

$$
X:=-Z^{-1}(\alpha E-\bar{\alpha} A)^{-1}
$$

that is clearly invertible. To show that $X$ is Hermitic we subtract (139) multiplied by $\alpha$ from (138) multiplied by $\bar{\alpha}$ and get

$$
X^{-1}=(\bar{\alpha} A-\alpha E) Z=Z^{*}\left(\alpha A^{*}-\bar{\alpha} E^{*}\right)=X^{*} .
$$

It only remains to show (128). We rewrite the left-hand side of (128) successively as

$$
\begin{gathered}
\frac{1}{2}\left[\left(\alpha A^{*}+\bar{\alpha} E^{*}\right) X(\alpha E-\bar{\alpha} A)+\left(\bar{\alpha} E^{*}-\alpha A^{*}\right) X(\alpha E+\bar{\alpha} A)\right]-C^{*} J C \\
\stackrel{(141)}{=}-\frac{1}{2}\left[\left(\alpha A^{*}+\bar{\alpha} E^{*}\right) Z^{-1}+Z^{-*}(\alpha E+\bar{\alpha} A)\right]-C^{*} J C \\
\stackrel{(138)-(139)}{=}-Z^{-*} B D^{-1} C-C^{*} J C \stackrel{(140)}{=} 0
\end{gathered}
$$

which ends this part of the proof.

If. We show that if (127)-(129) hold for an invertible Hermitic matrix $X$ then $M(z)$ is $J$ all-pass. Starting with (126) we get a realization of

$$
M^{*}\left(\frac{1}{\bar{z}}\right) J M(z)=\left[\begin{array}{cc|c}
E^{*}-z A^{*} & (\alpha-\bar{\alpha} z) C^{*} J C & (\alpha-\bar{\alpha} z) C^{*} J D \\
O & A-z E & (\alpha-\bar{\alpha} z) B \\
\hline B^{*} & D^{*} J C & D^{*} J \bar{D}
\end{array}\right] .
$$

Let

$$
Q:=\left[\begin{array}{cc}
I & \left(\alpha A^{*}-\bar{\alpha} E^{*}\right) X \\
O & I
\end{array}\right], \quad Z:=\left[\begin{array}{cc}
I & X(\bar{\alpha} A-\alpha E) \\
O & I
\end{array}\right] .
$$

Performing an equivalence state-space transformation with $Q$ to the left and $Z$ to the right we obtain

$$
M^{*}\left(\frac{1}{\bar{z}}\right) J M(z)=\left[\begin{array}{cc|c}
E^{*}-z A^{*} & O & O \\
O & A-z E & (\alpha-\bar{\alpha} z) B \\
\hline B^{*} & O & D^{*} J \bar{D}
\end{array}\right]
$$

where we have used $(128)$ and $(129)$ to get that the $(1,2),(1,3)$ and $(3,2)$ entries are zero. Thus, removing the uncontrollable and unobservable part of (145) we finally get

$$
M^{\sim}(z) J M(z)=D^{*} J D
$$

from where with (127) we conclude that $M(z)$ is $J$ all-pass. 